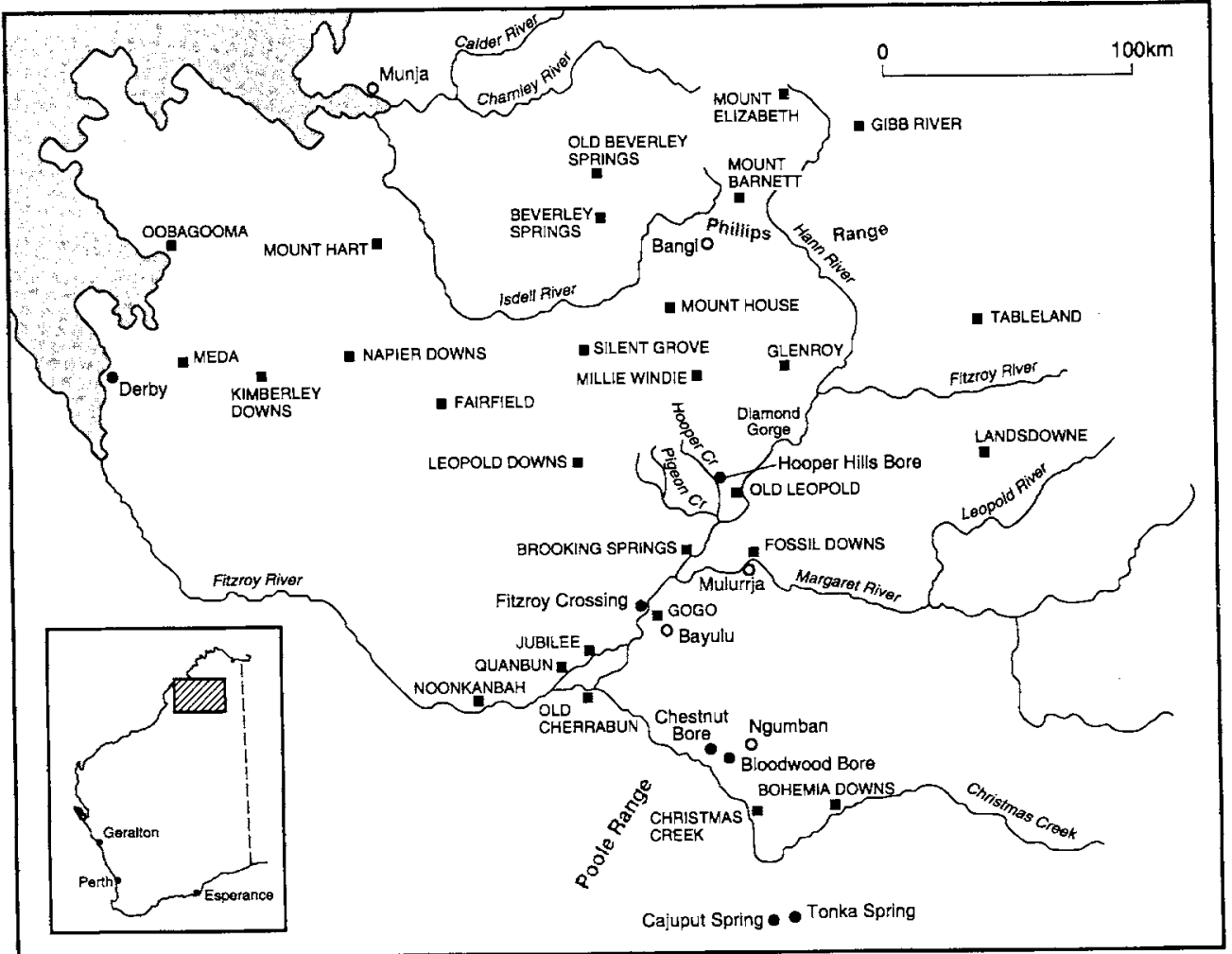

Central Kimberiey Region, Western Australia 


\title{
GOONIYANDI STORIES OF EARLY CONTACT WITH WHITES
}

\author{
Jack Bohemia, Bigfoot Jagarra, William McGregor \\ and Fossil Pluto
}

\section{PART I}

\section{TEXT 1: EARLY CONTACT WITH THE WHITES 1}

\section{By Fossil Pluto}

[Recorded at Fossil Downs, 1966, by Howard Coate. ${ }^{2}$

Transcribed and translated by Howard Coate and Fossil Pluto, Fossil Downs, 1966; retranscribed and retranslated by William McGregor and George Nayindu, Fitzroy Crossing 1980.]

thiwa, niyaji thangarndi wini jaminyinhingi

jaminyingga; goowajjangi

jaminyingga goowajjayi, nd ngoorroo

ngaboongga, goowajnga ngarragi jaminyi

malngarri wardbirri ngamoo, mangarri;

ngarramawinbirra whites, this story is just from my grandfather

my grandfather; told it

my grandfather told the story, and his

father, he told my grandfather

when the white people came, (they) didn't; know about them

Jack Bohemia was born on Old Bohemia Downs Station around the turn of the century. He worked for many years as a stockman on that station and a number of others in the region, and from 1938 until the 1970s as a tracker for the Fitzroy Crossing police. He is best known for his involvement in the apprehension of Banjo, who allegedly killed two white men on Billiluna station in 1922. In 1972 he was awarded the British Empire Medal for thirty two years service to the Western Australian police and community. Jack Bohemia is currently working with William McGregor on producing a book of his experiences as a police tracker (Nyibarri: Kimberley tracker).

Bigfoot Jagarra, a contemporary of Jack Bohemia's, was born on Fossil Downs station, where he worked for most of his life as a stockman. He now lives in Mulurrja, a community on the Margaret River some twenty kilometres upriver from Fitzroy Crossing. Bigfoot Jagarra is widely regarded as the most knowledgable Gooniyandi lawman alive today.

William McGregor holds an ARC Fellowship in Linguistics at University of Melbourne. He has made a depth study of Gooniyandi (published in 1990), and is currently engaged in writing an encyclopaedic grammar and dictionary of Nyulnyul, and shorter sketch grammars of Warrwa, Unggumi, Yawijibaya, Umiida, Unggarrangu and Gunin.

Fossil Pluto (deceased) was a highly regarded GooniyandiKija lawman who lived on Fossil Downs station.

1 Gooniyandy is a traditional Australian language spoken by one hundred or so Aborigines living in Fitzroy Crossing region of the Kimberley, Western Australia (see McGregor 1990 for a descriptive grammar). The texts below are represented in accordance with the system I have employed elsewhere - see e.g. McGregor 1988 and Bohemia \& McGregor 1991 - except that, for considerations of space, a columnar rather than facing page layout has been employed.

The orthography employed here is almost identical with the latest variant of the practical orthography chosen by speakers of the language. The main difference is that retroflection is represented by an $r$ preceding $d, n$, or $l$. (retroflection is not represented in the practical orthography).

2 We would like to thank Howard Coate for generously making both the tape recording and the original transcription and translation of this text available to us, and for permission to use it in this paper. 
milayawinbirra

dimana milawirra, dimana niyi daarri thiminyjangarri migawirrinhi

mamooyoo dimana tharra

marrimbala goowajbirra, malngarri ngambirri migawirrawirrangi gilimboormgamanyi

mangarri malawinbirra ngamoo

ngimdanyi waawabjiwinbirra, malngarri ngarloongarloo waabawirri

niyajinhingi, bagiwirri, maningga, lanygiyanyali woodijbinbirra jaminyi ngarragingga, niyaji thangarndi goowajji, nga:moo ngirnda

malngarri gardbilawinbirra

mangarri yaningi, winhi ganginybinbirra

daarri thiminyjangarri migawirrinhi niyaji dimanayoo

dimana, malngarringga rayidimjawirra

marrimbala, gilimboormgama, gilimboormgarna goowajbinbirra malngarri

woodijgilawinbirra miga limbaga moowa gardbiwinbirra yarrangi boolgawoolga, ngamiya

yoowooloo, migawirriwirrangi, daarri, yilbaya thirrimili warangbirri

malngarringga riwi ngimda moodbirra, yoowoolooyoo riwi

niyaji ganginybinbirra, ngaandi, yaanyabinyi warangjawirri

migangga gardbilawinbirra ngimdigangarriya limba yaanyangga thirrimilingga warlibirri ngirnda gindiwa, yilba, jibirri, boorroonggoo ngimda, bandarangarri, mayalnga

niyinhingi, jaroongjawindi, mayalnga gooji balwalwayawoorra niyaji, miganhingi

goomboo yaabjangga maaralmawinbirra gardbiwinbirra yarrangi

niyajinhingi thirrinygoowawirmi

woodijbinbirra

mangarri ngarramawinbirra

wila joomanygarra warangbirri yoowooloo they saw them

they saw horses, but they thought they were spirits of dogs

the horse was a dog spirit

they caled it a grass country devil, and white people again they called them dwellers of the long grass

they had never seen them before

they sniffed around this way, the white men smelt different

they, they camped, the night, and speared them during the middle of the day my grandfather, told me this story, before

they killed white people

not now, they were ignorant of them (before)

they thought that the horse was the spirit of a dog

horses, which the white people were riding

grass country devil, and long grass dweller, they called white people long grass dwellers

they speared only the policemen who killed our old people, in the old days

Aborigines, thought about them (the police), mistakenly, that they were very wild

white people tramped the country, Aboriginal country

the police were ignorant, in a body, the Aborigines were sitting on the other side whole

like that all the wild policemen killed Aborigines

up this river, for good, and down, north this, bandarangarri Margaret River

then, he covered, Margaret River the bones of the dead people lie about everywhere, because of that others stole women they killed our forebears then they got wild and speared them

they didn't know about them then Aborigines were $\mathrm{OK}$ 


\section{GOONTYANDI: EARLY CONTACT WITH WHITES IN THE KIMBERLEY}

malngarrinyali yaa wardbirriwirrangi, walawalaji

walajiwinbirra

ngiwayi jibirri, gindiwa, boorroonggoo ngirnda miga

yaningi joornanygarra malngarri waranggoorroo joornanygarra waranggoorroo, mangarri gardboowoorra, yoowooloo

wilawoo ngara, ngarawirili, malngarri

mangarri ganginygarrra

wini yaabjangga, goombooga wardbirriwirrangi malngarriyirra, thiwamoowa gilbajawarrawirrangi

yalawa ngarlarrayawoorraminhi

mangarri balajinhingi, yiganyinhing $i$ ward jawoorroo white people again went to them, looking for a fight

they were looking for a fight with them south downstream, upstream, north this way

today the white people are good, they are good, they don't kill Aborigines

now, they are quiet, whites

'this time we don't do something to do with relatives

should some, women go with white men, we find only part Aboriginal children with them

the little one lies on his back

its not from being sent to the whitemen, they went sneakingly to them

\section{TEXT WITH INTERLINEAR TRANSLATIONS ${ }^{3}$}

$\begin{array}{lllll}\text { thiwa, niyaji thangarndi } & \text { wini jaminyi } & - \text { nhingi } \\ \text { red } & \text { this } & \text { word } & \text { only grandfather } & -\mathrm{ABL}\end{array}$

'Whites. This story is just from my grandfather.' (1)

$\begin{array}{llll}\text { jaminyi } & \text {-ngga; } & \text { goowaj } & \text {-jangi } \\ \text { grandfather } & \text {-ERG } & \text { name } & \text {-he:caught:it }\end{array}$

'My grandfather told it.' (2)

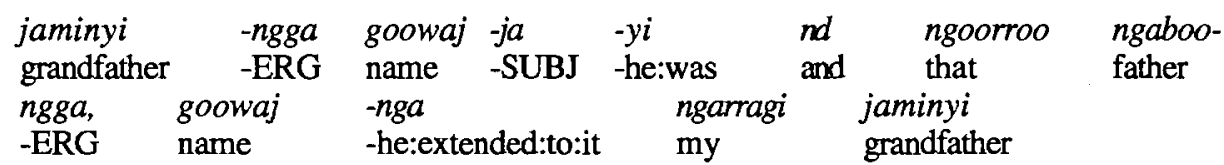

'My grndfather told the story, and his father told him.' (3)

$\begin{array}{lllllll}\text { malngarri } & \text { ward } & \text {-birri } & \text { ngamoo, } & \text { mangarri, } & \text { ngarma } & \text {-ma } \\ \text { white:person } & \text { go } & \text {-they:were } & \text { before } & \text { not } & \text { know } & \text {-IND }\end{array}$

-winberra

-they:extended-to-them

'When the white people came, no one knew about them.' (4)

mila -ya -winbirra

see -LOC -they:extended:to:them

'They saw them.' (5)

3 The following abbreviations are used in the interlinear translations: ABL - ablative; CHAR - characterised by; COMIT - comitative; DAT - dative; DEF - definite; DW - dweller of or inhabitant of niche; EMP emphatic; ERG - ergative; ETC - etcetera; FACT - factive mood; INCL - including; IND - indefinite, identity not known; IT - iterative; LOC - locative; PRE - prelative; PL - 0 plural; PROG - progressive aspect; REP - repetition, again; SEM - simulative; and SUBJ - subjunctive mood. For discussion of these grammatical categories, see McGregor 1990. A questionmark in the interlinear gloss line indicates that the meaning of the word or morpheme is not known. 


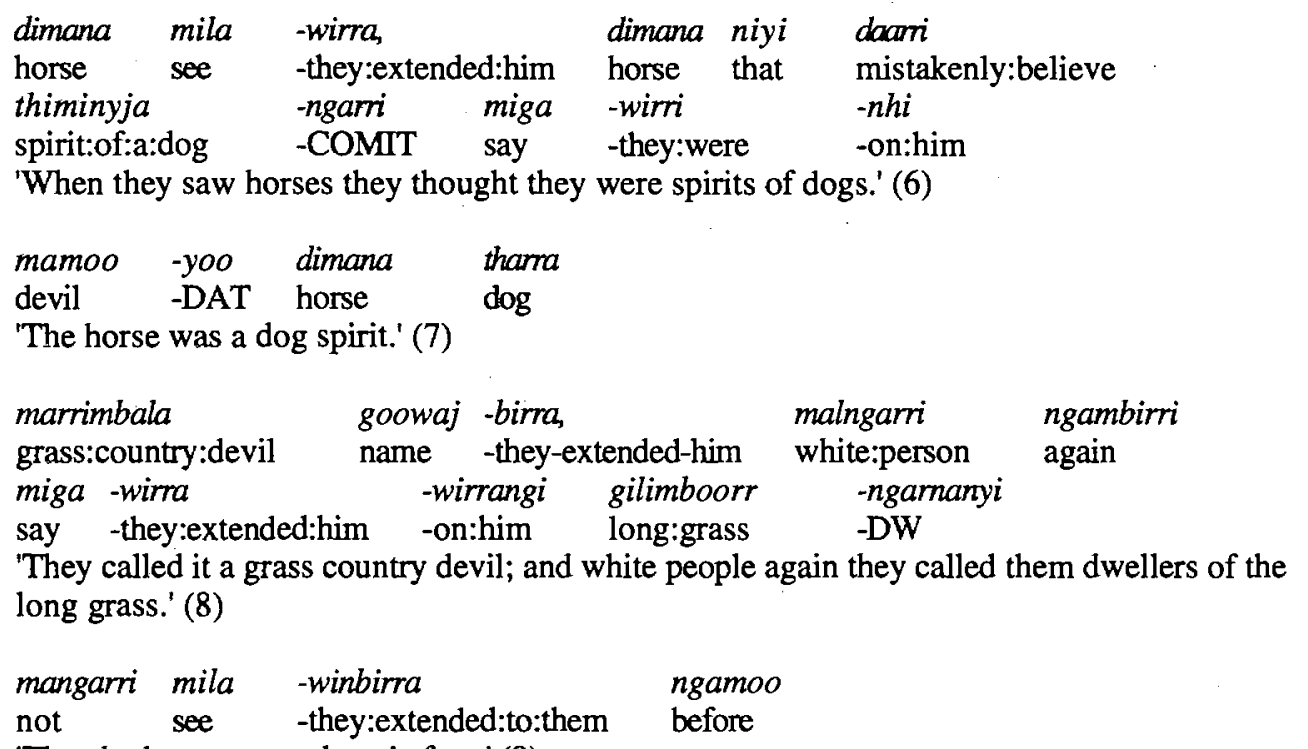

'They had never seen them before.' (9)

$\begin{array}{lllll}\text { ngimdanyi } & \text { waawab } & -j i & \text {-winbirra, } & \text { malngarri } \\ \text { this:way } & \text { smell } & \text {-IT } & \text {-they:extended:to:them } & \text { white:person } \\ \text { ngarloongarloo } & \text { waab } & -a & \text {-wirri } & \\ \text { different:smell } & \text { smell } & \text {-PROG } & \text {-they:were } & \end{array}$

'They sniffed around this way; the white men smelt different.' (10)

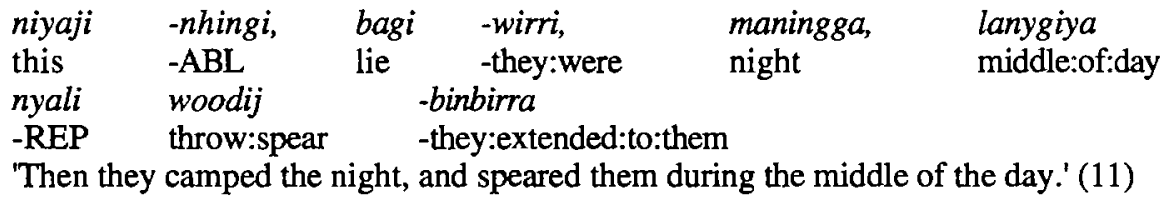

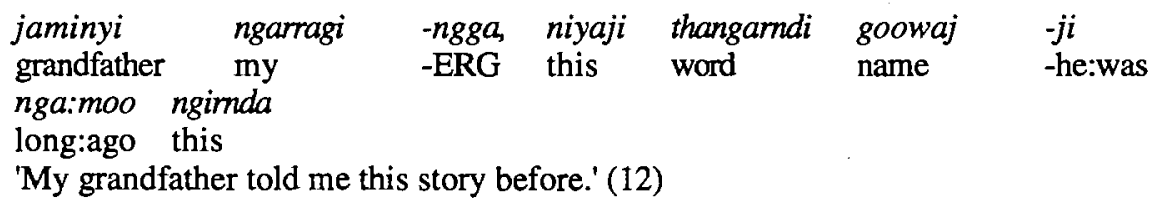

$\begin{array}{lllll}\text { malngarri } & \text { gard } & -b i & -l a & \text {-winbirra } \\ \text { white:person } & \text { hit } & -\mathrm{IT} & -\mathrm{FACT} & \text {-they:extended:to:them }\end{array}$

'They killed white people.' (13)

$\begin{array}{llll}\text { mangarri } & \text { yaningi, } & \text { winhi ganginy } & \text {-binbirra } \\ \text { not } & \text { now } & \text { only ignorant } & \text {-they:extended:to:them }\end{array}$

'Not now; they were ignorant of them (before).' (14) 


\section{GOONIYANDI: EARLY CONTACT WITH WHITES IN THE KIMBERLEY}

$\begin{array}{llllll}\text { daami } & \text { thiminyja } & \text {-ngarn } & \text { miga } & \text {-wirri } & \text {-nhi } \\ \text { mistakenly:believe } & \text { spirit:of:a:dog } & \text {-COMIT } & \text { say } & \text {-they:were } & \text {-on:him } \\ \text { niyaji dimana } & \text {-yoo } & & & & \\ \text { this horse } & \text {-DAT } & & & & \end{array}$

'They thought that the horse was the spirit of a dog.' (15)

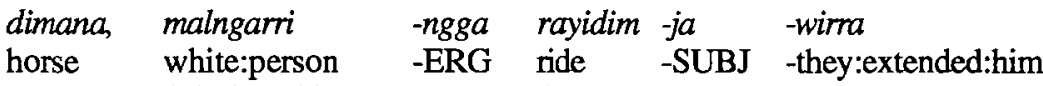

'Horses, which the white people were riding.' (16)

$\begin{array}{llllll}\text { marrimbala, } & \text { gilimboorr } & \text {-ngarna, } & \text { gilimboorr } & \text {-ngama } & \text { goowaj } \\ \text { grass:country:devil } & \begin{array}{l}\text { long:grass } \\ \text { malngarri }\end{array} & \text {-DW } & \text { long:grass } & \text {-DW } & \text { name } \\ \text {-binbirra } & \begin{array}{l}\text { malne } \\ \text {-they:extended:to:them }\end{array} & \text { white:person } & & & \end{array}$

'Grass country devil and long grass dweller; they called white people long grass

dwellers.' (17)

\begin{tabular}{|c|c|c|c|c|c|}
\hline $\begin{array}{l}\text { woodij } \\
\text { throw:spear }\end{array}$ & $\begin{array}{l}\text {-gila } \\
\text {-FACT }\end{array}$ & & $\begin{array}{l}\text {-winbirra } \\
\text {-they:extended:to:them }\end{array}$ & $\begin{array}{l}\text { miga } \\
\text { like:that }\end{array}$ & $\begin{array}{ll}\text { limba } & -g a \\
\text { policeman } & - \text { ERG }\end{array}$ \\
\hline $\begin{array}{l}\text {-moowa } \\
\text {-only }\end{array}$ & $\begin{array}{l}\text { gard } \\
\text { it }\end{array}$ & $\begin{array}{l}-b i \\
-\mathrm{IT}\end{array}$ & $\begin{array}{l}\text {-winbirra } \\
\text {-they:extended:to:them }\end{array}$ & $\begin{array}{l}\text { yarrangi } \\
\text { our }\end{array}$ & $\begin{array}{l}\text { boolgawoolga } \\
\text { old:men }\end{array}$ \\
\hline
\end{tabular}

ngami -ya

before $-\mathrm{LOC}$

'They speared only the policemen who killed our old people, in the old days.' (18)

$\begin{array}{llllll}\text { yoowooloo, miga } & \text {-wirri } & \text {-wirrangi, daari, } & \text { yilbaya } \\ \text { man } & \text { say } & \text {-they:were } & \text {-on:them } & \text { mistakenly:believe } & \text { policemen } \\ \text { thirri } & \text {-mili } & \text { warang } & \text {-birri } & & \\ \text { wild } & \text {-CHAR } & \text { sit } & \text {-they:were } & & \end{array}$

'Aborigines thought that the police were very wild.' (19)

$\begin{array}{llllll}\text { malngarri } & \text {-ngga } & \text { riwi } & \text { ngimda } & \text { mood } & \text {-birra, } \\ \text { white:person } & \text {-ERG } & \text { country } & \text { this } & \text { squash } & \text {-they:extended:him } \\ \text { yoowooloo } & - \text { yoo } & \text { riwi } & & & \\ \text { man } & \text {-DAT } & \text { country } & & & \end{array}$

'White people tramped the country, Aboriginal country.' (20)

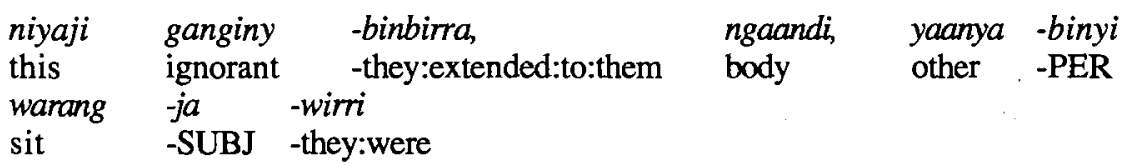

'The police were ignorant; the Aborigines were sitting on the other side whole.' (21) 


\section{ABORIGINAL HISTORY 1993 17:1}

\begin{tabular}{|c|c|c|c|c|c|c|c|c|}
\hline $\begin{array}{l}\text { miga } \\
\text { like:that }\end{array}$ & & & $\begin{array}{l}\text { gard } \\
\text { hit }\end{array}$ & $\begin{array}{l}-b i \\
-I T\end{array}$ & $\begin{array}{l}-l a \\
-F A C T\end{array}$ & $\begin{array}{l}-x \\
-t\end{array}$ & $\begin{array}{l}\text { zbirra } \\
\text { y:exte }\end{array}$ & d:to:them \\
\hline $\begin{array}{l}\text { ngirndi } \\
\text { this }\end{array}$ & $\begin{array}{l}-g a \\
-E R G\end{array}$ & $\begin{array}{l}\text {-ngarriya } \\
\text {-INCL }\end{array}$ & & $\begin{array}{l}\text { limba } \\
\text { policeman }\end{array}$ & $\begin{array}{l}\text { yaanya } \\
\text { other }\end{array}$ & $\begin{array}{l}-n g g a \\
-E R G\end{array}$ & $\begin{array}{l}\text { thirri } \\
\text { wild }\end{array}$ & $\begin{array}{l}\text {-mili } \\
\text {-CHAR }\end{array}$ \\
\hline
\end{tabular}

'Like that, all the wild policemen killed Aborigines.' (22)

warlibirri ngimda gindiwa, yilba, jibirri, boorroonggoo

river this upstream forever downstream north

ngimda, bandarangarri mayalnga

this (a very short river) Margaret River

'Up this river, for good, and down north, this Margaret River.' (23)

niyi -nhingi, jaroong -ja -windi, mayalnga

that -ABL ? -SUBJ -he:got Margaret River

'Then, he covered Margaret River.' $(24)^{4}$

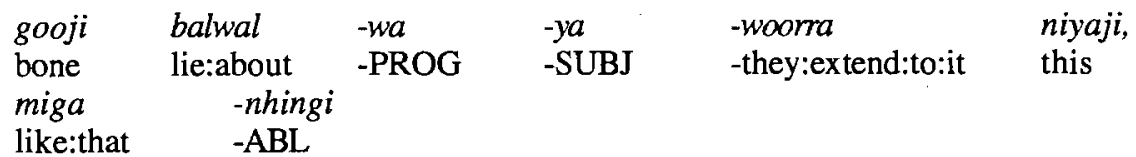

'The bones of the dead people lie about everywhere because of that.' (25)

$\begin{array}{llllll}\text { goomboo } & \text { yaabja } & \text {-ngga } & \text { maaral } & -m a & \text {-winbirra } \\ \text { woman } & \text { other } & -\mathrm{ERG} & \text { steal } & -\mathrm{NND} & \text {-they:extended:to:them }\end{array}$

'Others stole women.' (26)

gard -bi -winbirra yarrangi

hit -IT -they:extended:to:them our

'They killed our forebears.' (27)

niyaji -nhingi thirriny -goowa -wirrmi woodij

this -ABL get:wild -PROG -they:effected throw:spear

-binbirra

-they:extended:to:them

'Then they got wild and speared them.' (28)

mangarri ngara -ma -winbirra

not know - $\mathbb{N D}$-they:extended:to:them

'They didn't know about them.' (29)

$\begin{array}{lllll}\text { wila } & \text { joornanygarra } & \text { warang } & \text {-birri } & \text { yoowooloo } \\ \text { finish } & \text { good } & \text { sit } & \text {-they:were } & \text { man }\end{array}$

'Then Aborigines were OK.' (30)

4 The meaning of the term jaroong - is unclear. From the context, however, the sentence would seem to suggest that the policeman went along the Margaret River killing Aborigines. 


\section{GOONIYANDI: EARLY CONTACT WITH WHITES IN THE KIMBERLEY}

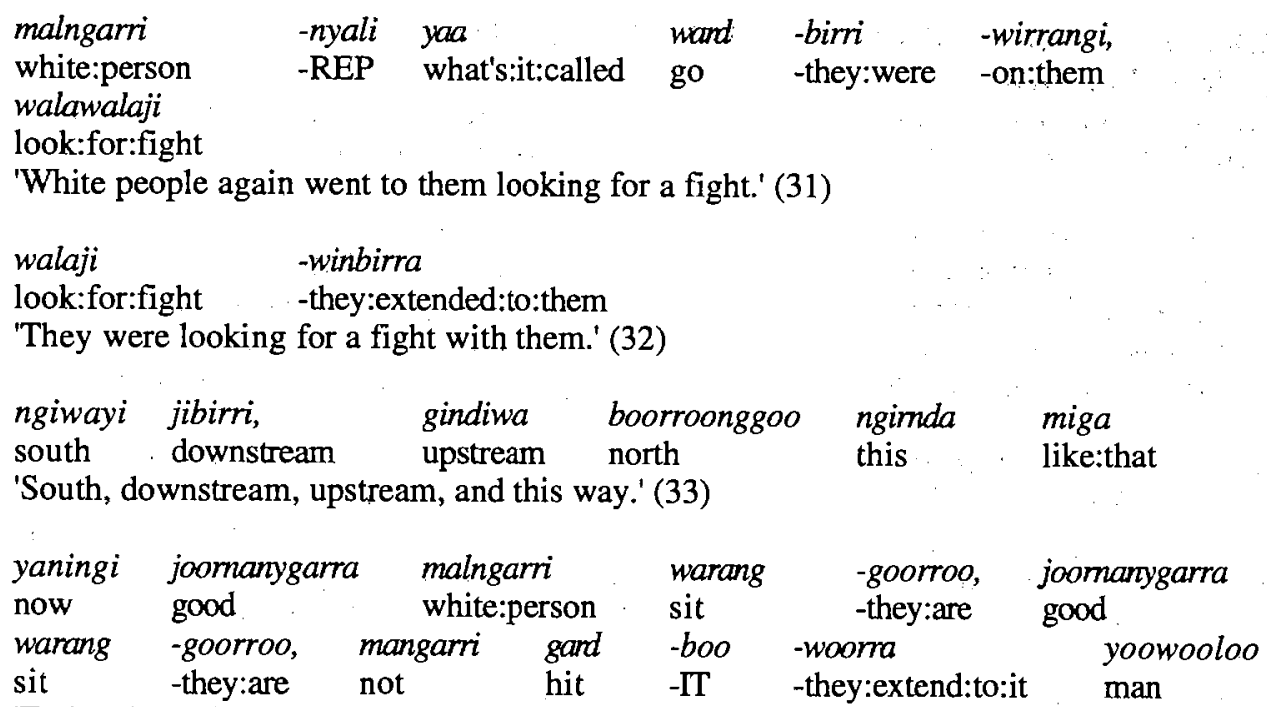

'Today the white people are good; they don't kill Aborigines.' (34)

$\begin{array}{lll}\text { wilawoo ngara, ngarawirili, } & \text { malngarri } \\ \text { finish quiet quiet } & \text { white:person } \\ \text { 'The country is quiet now.' (35) } & \end{array}$

'The country is quiet now.' (35)

$\begin{array}{lll}\text { mangarri } & \text { ganginy } & \text {-garra } \\ \text { not } & \text { ignorant } & \text {-we:extend:to:them }\end{array}$

' "This time we don't do something to do with relatives." ' $(36)^{5}$

$\begin{array}{llllllll}\text { wini yaabja } & \text {-ngga, } & \text { goomboo } & \text {-ga } & \text { wand } & \text {-birri } & \text {-wirrangi } \\ \text { only other } & \text {-ERG } & \text { woman } & \text {-ERG } & \text { go } & \text {-they:were } & \text {-on:them } \\ \text { malngarri } & \text {-yirra, } & \text { thiwa } & \text {-moowa } & \text { gilba } & \text {-ja } & \text {-warra } & \text {-wirrangi } \\ \text { white:person } & \text {-ALL } & \text { red } & \text {-only } & \text { find } & \text {-SUBJ } & \text {-we:will:be } & \text {-on:them }\end{array}$

'When some women go with white men, we find only part Aboriginal children with them.' (37)

$\begin{array}{lllll}\text { yalawa } & \text { ngarlarra } & - \text { ya } & - \text { woorrami } & -n h i \\ \text { close } & \text { back } & \text {-SUBJ } & -? & \text {-on:him }\end{array}$

'The little one lies on his back.' (38)

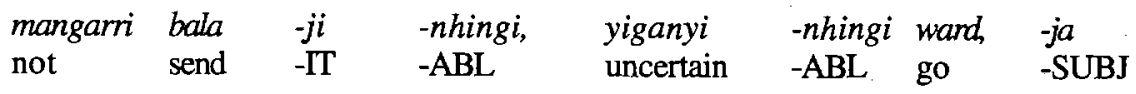

-woorroo

-they:are

'We didn't send them to the whitemen, they went sneekingly to them.' (39)

5 From the meaning of the individual words in this sentence a meaning like 'We are not ignorant' is suggested. This also fits with the context. However, the Gooniyandi speaker provided the translation given here, the interpretation of which remains obscure. 


\section{TEXT 2: EARLY CONTACT WITH THE WHITES By Jack Bohemia}

[Recorded at Fitzroy Crossing, 1982, by William McGregor, and transcribed and translated shortly later by William McGregor with the assistance of Jack Bohemia.]

bidi ngirnda, gimangarna yoowooloo wajbaliyoo mangarri binarri wamba wajbali bij;ngarniwirrangi aa, thiwa, goowajbinbirra thiwa ngirnda ngoonyoongama bijbirrarni, thiwa

mangarri binarri wajbaliyoo

yawarda, milawirra

yawarda, goowajbirra yingi, maroonbala

yingi goowajbirra marrinbala

niyinhingi, niyinhingi, maroorrwinbini, wajbalingga

warrgoom, ngangbindi

mangarri yingglijjoo binarri, winhi warrgoombirri

manyiyoo, mangarri binarri; balawayoo, thamba diyoo marlami

wini ngabbirra

ngoorndoo, ngoorndoongoornoongga, gardiyangga migamiwirrangi manyi ngimdaji ngabbinggirra

milawirra:, drau drawu ngara ,..., goolooljiwirra; ngabbirr goolooljiwirra, ngabbirra:

aa jimandinginyji, migawinmi, jimandigawoo

manyi ngimda jimandi

ngabbirra garndiwangoorroonggarni

garndiwangoorroonggarmi ngabbirra

binarriwirri niyaji

manyi ngabbirra:, wili

dinyooloo, di, nalija, joowa, yoodoongarni; majangga

ngoorloorloogbirra yaa, jimandi girli, migawirni

jimandawoo, garndiwangoorroo migawirri ngirrinyjila girli ngabbarra wimi

ngabbirra: wili

binarriwawirrima, ngamoo; wa. wangarla warangbirri

mangarri manyiyoo binarri, wajbaliyoo mangarri binarri, wirni warangbirri wangarla

ngabbirra: wila these people, were bushmen

(they) didn't know of white people

later the white people came to them

um, reds, they called them reds

'where are these people from, the whites?'

(they said)

they didn't know about white people

horses, they saw them

horses, they called by the name 'maroonbala' they called it 'marrinbala'

then, then, they got them together, white people

work, they gave them

they didn't know English, they just worked

as for food, they didn't know; about white man's flour, damper, tea, nothing

they just ate it

what, what's it called, the white people told them 'eat this food'

they looked, try try, ..., they tried it; they ate it, they tried it; and ate it

'ah it's good!', they said, 'good!'

'this food it good'

then they all ate it

then everyone ate it

they learnt about these (foods)

they ate the food, and finished it

tea etc, tea, tea, ? the boss put out (for them)

they drank and drank it, 'ah good one,' they said

'good!', they all said

'we'll eat it hungry'

they ate it up

now they knew, before; they were ignorant

they didn't know about (white people's) foods, they didn't know about white people, they were just ignorant

they ate it up 


\section{GOONIYANDI: EARLY CONTACT WITH WHITES IN THE KIMBERLEY}

binarrigbindi, bindimi, wajbalingga

binarrig;binmi

binarrigbinmi

binarrima warangbirn

warrgoom ngangjiwina:: wili

binarriwawiri

wamba, ngoomdoongoornoo, wagan gilbawirri, booloomaningga rirrnga

ngirnda jajiwirri rirrwirra migami, migawinmi

thinga lajgiri

baarri goowajbirra bidingga baarri

mangarri wagan goowajbirra, baarn

booloomani, wila booloomani yingi

goowajbirra

wagan baarn, goowajbirra

thinga, gilbawirri

lajgoowaarni ngilayani

niyinhingi, binarriwawirrirna

binarriwawirri:: wila

yoowami yoowami, dardigirmiwirra

yingglij, dard; dardigirmiwirra

jijagbirri, thigiyigi

wamba, binarriyawirn, gardiyangga le;

ngangbindi yawarda

ngirnda lajawinggirra

lajawirra:: wila

binarriwawirri, goordangoornoonga lajawirra

yilba binarriwirrima

ngoorndoongoornoo; we;

booloomani, doownga, majangga, yingi Bill

Richardson

booloomani bayimjingi, Queenslannhingi

ngilmangi wardbirra

niyajiya breedim, growimupbirra, booloomani

$e:$, niyiyarndi yoowooloo binarriwirrima, boolgawoolga

binarriwawirri, binarriwawirrima:

lajalajawirra booloomani:: wilajjiwirra

brand

brandimbirra yardja

yard. ngaragbirra

ngaragnga, wajbali, wajbali,

boorlooboowirra

wajbalingga; binarrikjiwina

yard ngaragbirra

ngaragbirra:: wili

yard, booloomaniyoo they taught him, them, the white people taught; them

they taught them

then they knew

he gave them work finish

they were knowledgeable

later, what-cha-ma-call-it, they noticed wagons, bullocks pulling them

'I don't know what it is pulling it' he said ... they said

'the track lies'

they called it 'baarri'

they didn't call it a wagon, but a 'baarri'

the bullock, yes they called it 'booloomani'

wagons 'baarri', they called them

tracks, they found them

it goes east

then, they knew now

they knew OK

bit by bit, they misunderstood them

English, they misunderstood it

they spoke, just a little

later on, they learnt, and the white people gave them horses

'ride this!'

they rode it OK

they learnt, to ride horses

they knew now

what-cha-ma-call-it?; um;

cattle, he got them, the boss, called Bill

Richardson

he bought the cattle, in Queensland

they brought them

there they breed, they grow up, cattle

ah, those old people were knowledgeable now, the old people

they knew now, they knew now how to ride bullocks about brand ...

they branded them in the yard

yards, they made them

they made it, the whites, the whites, they

followed them

the white man; taught them

they made the yard

they made it and finished it

a yard, for cattle 


\section{ABORIGINAL HISTORY 1993 17:1}

goobardiya, ngila, joonyoo, niyajiya yard

ngaragbinmi, mayariya

yoo niyajiyarndi garndiwirri

niyajiyawoo; brandimbirra, booloomani

brandimbirra:: wili

ngarrjawinmirna, booloomani

ngarriawawinmi

niyinhingi, yaanyaya m. gamdiwirrja ya, nga ngarloorrja ya, garloorrja ya ngab; gamba

nyamani nyamani booloomanima, bullock nyamanyamani

balayoo; balayingi, majangga balayingi jibirri

drovim wardbirra, derbyyayi

yamadiya, dagoornwirrarri

yamadi, yingi boat

niyajiya dagoorrwarriwirra

jibirri balayingi nyamaniyirra mayaroo

niyinhingi bal; balbirrawinmi

ngambirrinyali, jilyjarniwirra, booloomani

jilyyarniwirra lambalambardi::

brandngarringga

jilyyarniwirra:: wili

barranggaya

barranggabarranggawawani, wila gadbirrini gadbirrini wirnimi warangbirri at Goobardiya, and east, at Joonyoo, there they made yards, at the homesteads yes at both places

right there; they branded; the cattle they branded them and finished

they grew up a little now, the cattle grew up a little

then, after two, three, three years

the cattle were big then, big cattle

send; he sent it, the boss sent (word) downstream

they droved them, to Derby

in a boat, they put them

in a vessel, called a boat

they put them in there

the sent them down to the big city

then they ret; returned

again, they branded, cattle

they branded the little ones with brands

they branded them OK

in the build up to the wet

as it gets hot, they leave off work

they left work and took a break

\section{ORIGINAL TEXT WITH INTERLINEAR TRANSLATIONS}

bidi ngimda gima -ngama yoowooloo

they this bush -DW man

'These people were bushmen.' (1)

$\begin{array}{llll}\text { wajbali } & -y o o & \text { mangarri } & \text { binarri } \\ \text { white:people } & \text {-DAT not } & \text { know }\end{array}$

'They didn't know of white people.' (2)

wamba wajbali bij? -ngarni -wirrangi

later white:people emerge -it:emerged -on:them

'Later the white people came to them.' (3)

aa thiwa, goowaj -binbirra thiwa

aa red name -they:extended:to:them red

'Um, reds they called them, reds.' (4)

ngimda ngoonyoo -ngarna bij -birrarni, thiwa

this which -DW emerge -they:emerged red

' "Where are these people from, the whites?" (they said).' (5) 


\section{GOONIYANDI: EARLY CONTACT WITH WHITES IN THE KIMBERLEY}

$\begin{array}{llll}\text { mangarri } & \text { binarri } & \text { wajbali } & \text {-yoo } \\ \text { not } & \text { know } & \text { white:people } & \text {-DAT }\end{array}$

'They didn't know about white people.' (6)

yawarda, mila -wirra

horse see -they:extended:him

'They saw horses.' (7)

yawarda, goowaj -birra yingi, maroonbala horse name they:extended:him name horse

'They called horses "maroonbala".' (8)

yingi goowaj -birma marrinbala

name name -they:extended:him horse

'They called it "marrinbala".' (9)

niyi -nhingi, niyi -nhingi, maroor -winbini, wajbali

that $-\mathrm{ABL}$ that $-\mathrm{ABL}$ muster -he:hit:them white:people

-ngga

-ERG

'Then the white people got them together.' (10)

$\begin{array}{lll}\text { warrgoom, } & \text { ngang } & \text {-bindi } \\ \text { work } & \text { give } & \text {-he:caught:them }\end{array}$

'They gave them work.' (11)

$\begin{array}{lllllll}\text { mangarri } & \text { yingglij } & \text {-joo } & \text { binarri, } & \text { winhi } & \text { warrgoom } & \text {-birri } \\ \text { not } & \text { English } & \text {-DAT } & \text { know } & \text { only } & \text { work } & \text {-they:were }\end{array}$

'They didn't know English, they just worked.' (12)

$\begin{array}{lrlllll}\text { manyi } & -y o o, & \text { mangarri } & \text { binarri; balawa } & -y o o, & \text { thamba } \\ \text { vegetable:food } & \text {-DAT } & \text { not } & \text { know } & \text { flour } & \text {-DAT damper } \\ d i & \text {-yoo } & \text { marlami } & & & & \\ \text { tea -DAT nothing } & & & & \end{array}$

'They didn't know about white man's food, flour, damper, tea, nothing.' (13)

wini ngab -birma

only eat -they:extended:him

'They just ate it.' (14)

$\begin{array}{llllll}\text { ngoorndoo, } & \text { ngoorndoongoornoo } & -n g g a, & \text { gardiya } & \text {-ngga miga } \\ \text { someone } & \text { what's:it:called } & -\mathrm{ERG} & \text { white:person -ERG say } \\ \text {-mi } & \text {-wirrangi manyi } & \text { ngimdaji ngab -binggirra } \\ \text {-he:effected } & \text {-on:them vegetable:food this } & \text { eat } & \text {-you:pl:will:extend:to:it } \\ \text { 'What's it called, the white people told them "Eat this food." '(15) }\end{array}$


ABORIGINAL HISTORY 1993 17:1

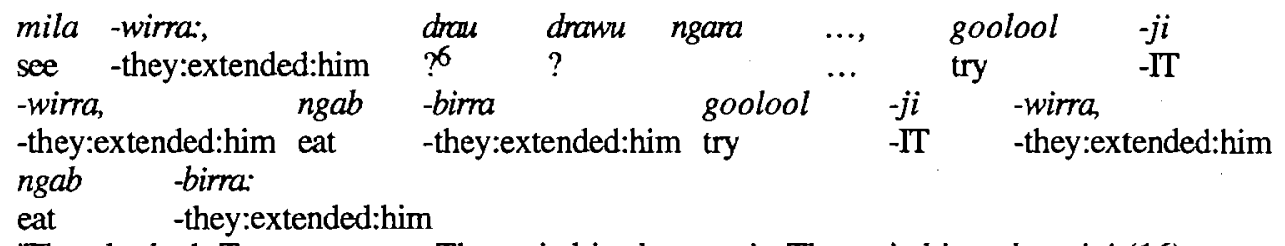

'They looked. Try, ... try, ... . They tried it; they ate it. They tried it and ate it.' (16)

$\begin{array}{lllllll}\text { aa jimandi } & \text { nginyji, } & \text { miga } & \text {-winmi, } & \text { jimandi } & \text {-gawoo } \\ \text { aa } & \text { good } & \text { EMP } & \text { say } & \text {-they:effected } & \text { good } & \text {-EMP }\end{array}$

" "Ah, it's good!", they said, "good!". (17)

$\begin{array}{lll}\text { manyi } & \text { ngimda } & \text { jimandi } \\ \text { vegetable:food } & \text { this } & \text { good }\end{array}$

" "This food it good." ' (18)

$\begin{array}{lllll}n g a b & -b i r r a & \text { garndiwangoorroo } & -n g g a & -m i \\ \text { eat } & \text {-they:extended:him } & \text { many } & - \text { ERG } & - \text { next }\end{array}$

'They they all ate it.' (19)

$\begin{array}{lllll}\text { gamdiwangoorroo } & -n g g a & -m i & n g a b & -b i r r a \\ \text { many } & \text {-ERG } & - \text { next } & \text { eat } & \text {-they:extended:him }\end{array}$

'Then everyone ate it.' (20)

binarri -wirri niyaji

know -they:went this

'They learnt about these (foods).' (21)

$\begin{array}{llll}\text { manyi } & \text { ngab } & \text {-birra: } & \text { wili } \\ \text { vegetable:food } & \text { eat } & \text {-they:extended:him } & \text { finish }\end{array}$

'They ate the food and finished it.' (22)

$\begin{array}{lllllll}\text { di } & \text {-nyooloo di, nalija, joowa, yoodoo -ngami } & \text { maja } & \text {-ngga } \\ \text { tea } & \text {-ETC } & \text { tea tea } & \text { ? } & \text { put } & \text {-he:extended to:it } & \text { boss }\end{array}$

'Tea, etc, tea, and ? the boss put out (for them).' (23)

ngoorloorloog -birra yaa, jimandi girli,

drink -they:extended:him what's:it:called good same

miga -wirri

say -they:were

"They drank and drank it, "Ah, good one," they said.' (24)

jimanda -woo, garndiwangoorroo miga -wirri

good -DEF many say -they:were

' "Good!", they all said.' (25)

6 It is not clear precisely what the speaker intended to say here. As Jane Simpson has pointed out, the most likely possibility is that $d r a u$ or $d r a w u$ is a version of the English word try. 


\section{GOONIYANDI: EARLY CONTACT WITH WHITES IN THE KIMBERLEY}

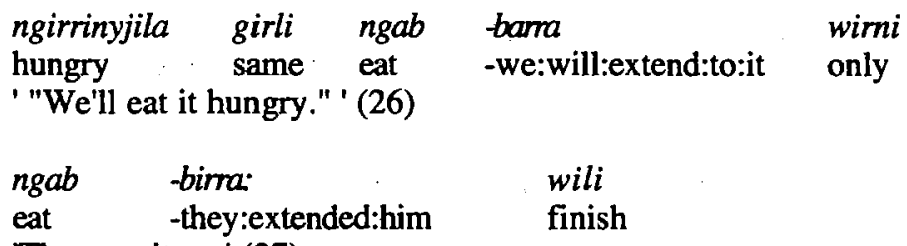

'They ate it up.' (27)

\begin{tabular}{|c|c|c|c|c|c|c|c|}
\hline $\begin{array}{l}\text { binarri } \\
\text { know } \\
\text {-birri } \\
\text {-they:wer }\end{array}$ & $\begin{array}{l}\text {-wa } \\
\text {-PROG }\end{array}$ & $\begin{array}{l}\text {-wirri } \\
\text {-they:were }\end{array}$ & $\begin{array}{l}-m a, \\
\text {-now }\end{array}$ & $\begin{array}{l}\text { ngamoo. } \\
\text { before. }\end{array}$ & $\begin{array}{l}\text { wa. } \\
\text { wa }\end{array}$ & $\begin{array}{l}\text { wangarla } \\
\text { ignorant }\end{array}$ & $\begin{array}{l}\text { warang } \\
\text { sit }\end{array}$ \\
\hline
\end{tabular}

$\begin{array}{lllllll}\text { mangarri } & \text { manyi } & \text {-yoo binarri, wajbali } & \text {-yoo } & \text { mangarri } \\ \text { not } & \text { vegetable:food } & \text {-DAT } & \text { know white:people } & \text {-DAT } & \text { not } \\ \text { binarri, wirni warang } & \text {-birri } & \text { wangarla } & & \\ \text { know } & \text { only sit } & \text {-they:were ignorant } & & \end{array}$

'They didn't know about white people or their foods; they were just ignorant.' (29)

$\begin{array}{lll}\text { ngab } & \text {-birra: } & \text { wila } \\ \text { eat } & \text {-they:extended:him } & \text { finish }\end{array}$

'They ate it up.' (30)

$\begin{array}{lllll}\text { binarrig; } & \text {-bindi, } & \text {-bindimi } & \text { wajbali } & \text {-ngga } \\ \text { know } & \text {-he:caught:them } & \text {-they:effected:them } & \text { white:people } & \text {-ERG } \\ \text { binarrig; } & \text {-binmi } & & & \\ \text { know } & \text {-they:effected:him } & & \end{array}$

'They taught them; the white people taught them.' (31)

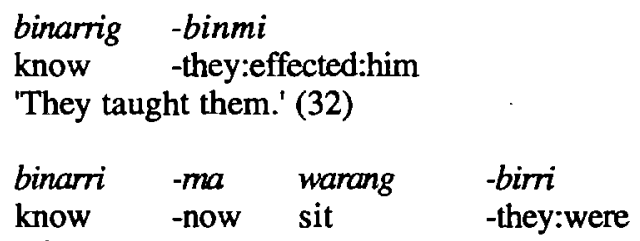




\section{ABORIGINAL HISTORY 1993 17:1}

wamba, ngoorndoongoornoo, wagan gilba

-wirri,

later what's:it:called wagon find

-they:caught:it

booloomani

-ngga rirr -nga

-ERG pull -he:extended:to:it

'Then what-cha-ma-call-it, they noticed wagons, bullocks pulling them.' (36)

$\begin{array}{llclll}\text { ngirnda } & \text { jaji } & \text {-wirri } & \text { nir } & \text {-wirra } & \text { miga } \\ \text { this } & \text { something } & \text {-I:do:not:know:what } & \text { pull } & \text {-they:extended:him } & \text { say } \\ \text {-mi, } & \text { miga } & \text {-winmi } & & & \\ \text {-he:effected } & \text { say } & \text {-they:effected } & & \end{array}$

' "I don't know what it is pulling it," he said ... they said.' (37)

thinga laj -giri

foot lie -he:is

' "The track lies." ' (38)

$\begin{array}{llllll}\text { baari } & \text { goowaj } & \text {-birra } & \text { bidi } & \text {-ngga } & \text { baarn } \\ \text { wagon } & \text { name } & \text {-they:extended:him } & \text { they } & \text {-ERG } & \text { wagon }\end{array}$

'They called it "baarri".' (39)

$\begin{array}{lllll}\text { mangarri } & \text { wagan } & \text { goowaj } & \text {-birra } & \text { baari } \\ \text { not } & \text { wagon } & \text { name } & \text {-they:extended:him } & \text { wagon }\end{array}$

'They didn't call it a wagon, but a "baarri".' (40)

booloomani, wila booloomani yingi goowaj -birra

bullock finish bullock name name -they:extended:him

'The bullock, yes, they called it "boloomani".' (41)

$\begin{array}{llll}\text { wagan baarri } & \text { goowaj } & \text {-birra } \\ \text { wagon } & \text { wagon } & \text { name } & \text {-they:extended:him }\end{array}$

'But they called wagons "baarri".' (42)

thinga, gilba -wirri

foot find -they:caught:it

'They found it's tracks.' (43)

laj -goowa -ami ngilayani

lie -PROG -it:falls east

'It goes east.' (44)

niyi -nhingi, binarri -wa -wirri -ma

that know -ABL -PROG -they:were -now

'Then they knew.' (45)

binarri -wa -wirri:: wila

know -PROG -they:were finish

'They knew, OK.' (46) 


\section{GOONIYANDI: EARLY CONTACT WITH WHITES IN THE KIMBERLEY}

\begin{tabular}{|c|c|c|c|c|}
\hline $\begin{array}{l}\text { yoowarni } \\
\text { one }\end{array}$ & $\begin{array}{l}\text { yoowarni, } \\
\text { one }\end{array}$ & $\begin{array}{l}\text { dandigirr } \\
\text { misunderstand }\end{array}$ & $\begin{array}{l}-m i \\
-\mathrm{IT}\end{array}$ & $\begin{array}{l}\text {-wirra } \\
\text {-they:extended:to:them }\end{array}$ \\
\hline \multicolumn{5}{|c|}{ 'They misunderstood English.' (47) } \\
\hline yingglij, & dand; & digirr & $-m i$ & -wirra \\
\hline English & dard & understand & $-\Gamma \mathrm{T}$ & -they:extended:to:them \\
\hline
\end{tabular}

'They misunderstood English.' (48)

jijag -birri, thigi -yigi

speak -they:were short -short

'They spoke just a little.' (49)

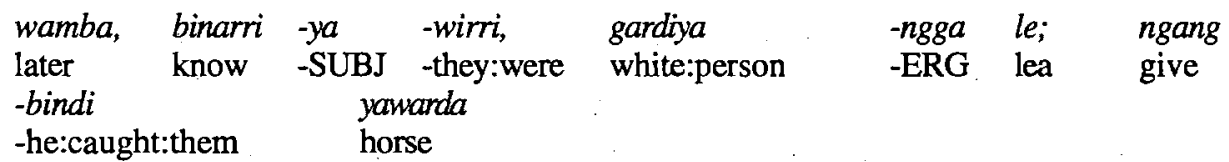

'Later on, they learnt, and the white people gave them horses.' (50)

ngimda laja -winggirra

this ride -you:pl:will:extend:to:it

' "Ride this!" ' (51)

$\begin{array}{lll}\text { laja } & \text {-wirra: } & \text { wila } \\ \text { ride } & \text {-they:extnded:him } & \text { finish }\end{array}$

'They rode it, OK.' (52)

$\begin{array}{llllll}\text { binarri } & - \text { wa } & \text {-wirri, } & \text { goordangoomoonga } & \text { laja } & \text {-wirra } \\ \text { know } & \text {-PROG } & \text {-they:were } & ? & \text { ride } & \text {-they:extended:him }\end{array}$

'They learnt to ride horses.' (53)

$\begin{array}{llll}\text { yilba } & \text { binami } & - \text { wirri } & -m a \\ \text { forever } & \text { know } & \text {-they:were } & \text {-now }\end{array}$

'They knew now.' (54)

ngoorndoongoomoo; we;

what's:it:called ?

'What-cha-ma-call-it?' (55)

booloomani, doow -nga maja -ngga yingi Bill

Richardson
bullock get -he:extended:to:it boss -ERG name

'He got cattle, the boss, called Bill Richardson.' (56)

booloomani bayim -jingi, Queenslan -nhingi

bullock buy -he:caught:it -ABL

'He bought the cattle in Queensland.' (57) 


\section{ABORIGINAL HISTORY $1993 \quad$ 17:1}

ngilmangi

from:the:east

ward

go

'They brought them.' (58) -birra

-they:extended:him

$\begin{array}{lllll}\text { niyaji } & -y a & \text { breedim, } & \text { growimup } & \text {-birra } \\ \text { this } & \text {-LOC } & & \text {-they:extended:him } & \text { booloomani } \\ \text { bullock }\end{array}$

'There they breed cattle.' (59)

e; niyi -yandi yoowooloo binarri -wirri -ma, boolgawoolga

ah that -PL man know -they:were -now old:men

'Ah, those old people were knowledgeable now.' (60)

binarri -wa -wirri, binarri -wa

know -PROG -they:were know -PROG -they:were -now ride

-laja -wirra booloomani:: wilaj -ji -wirra

-ride -they:extended:him bullock around -IT -they:extended:him

'They knew now, they rode bullocks about, brand ...' (61)

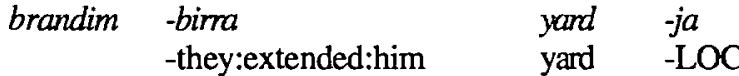

'They branded them in the yard.' (62)

yard ngarag -birra

yard make -they:extended:him

'They made the yard.' (63)

$\begin{array}{lllll}\text { ngarag } & \text {-nga, } & \text { wajbali, } & \text { wajbali, } & \text { boorlooboo } \\ \text { make } & \text {-he:extended:to:it } & \text { white:people } & \text { white:people } & \text { follow }\end{array}$

-wirra

-they:extended:him

'The white man made it, and they followed him.' (64)

wajbali $\quad$-ngga; binarrig $\quad-j i \quad$-wina

white:people -ERG know -IT -it:extended:to:them

'The white man taught them.' (65)

yard ngarag -birra

yard make -they:extended:him

'They made the yard.' (66)

ngarag -birra: $\quad$ wili

make -they:extended:him finish

'They made it and finished it.' (67)

yard booloomani -yoo

yand bullock -DAT

'A yard for cattle.' (68) 


\section{GOONIYANDI: EARLY CONTACT WITH WHITES IN THE KIMBERLEY}

goobardiya, ngila, joonyoo, niyaji -ya yaand ngarag Goobardiya east Joonyoo this -LOC yard make -binmi, -they:effected:him mayari $-y a$

'At Goobardiya and east at Joonyoo, there they made yards, at the homesteads.' (69)

$\begin{array}{llll}\text { yoo } & \text { niyaji } & -y a r n d i & \text { gamdiwirri } \\ \text { yes } & \text { this } & -\mathrm{PL} & \text { two }\end{array}$

'Yes, at both places.' (70)

niyaji -ya -woo; brandim -birra, booloomani this -LOC -DEF -they:extended:him bullock

'Right there they branded the cattle.' (71)

\begin{tabular}{|c|c|}
\hline randim & $\begin{array}{l}\text {-birma: } \\
\text {-they:extended:him }\end{array}$ \\
\hline
\end{tabular}

'They branded them, and finished.' (72)

$\begin{array}{lllllll}\text { ngarrja } & - \text { winmi } & -m a & \text { booloomani } & \text { ngarja } & \text {-wa } & \text {-winmi } \\ \text { little } & \text {-they:effected } & \text {-now } & \text { bullock } & \text { little } & \text {-PROG } & \text {-they:effected }\end{array}$ 'The cattle grew up a little.' (73)

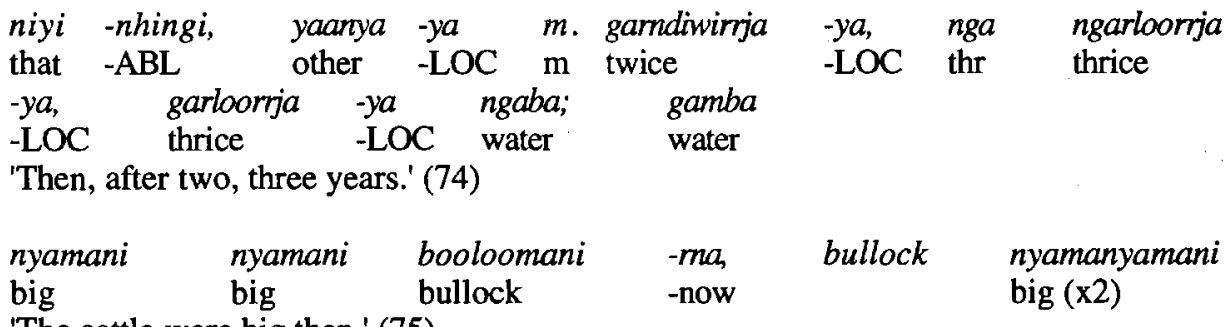

'The cattle were big then.' (75)

bala yoo; bala -yingi, maja -ngga bala -yingi

send ? send -he:caught:it boss -ERG send -he:caught:it

jibirri

downstream

'The boss sent word downstream.' (76)

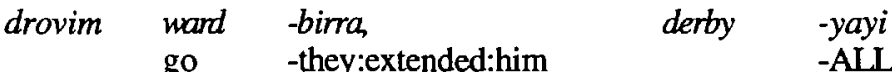

'They droved them to Derby.' (77)

yamadi $-y a, \quad$ dagoorr. wirrarri

vessel -LOC enter -they:put:it

'They put them in a boat.' (78)

yamadi, yingi boat

vessel name

'In a vessel called a boat.' (79) 
$\begin{array}{lllll}\text { niyaji } & -y a & \text { dagoor } & \text {-warri } & \text {-wirra } \\ \text { this } & - \text { LOC } & \text { enter } & \text {-IT } & \text {-they:extended:him }\end{array}$

'They put them in there.' (80)

$\begin{array}{llllll}\text { jibirri } & \text { bala } & \text {-yingi } & \text { nyamani } & \text {-yirra } & \text { mayaroo } \\ \text { downstream } & \text { send } & \text {-he:caught:it } & \text { big } & \text {-ALL } & \text { house }\end{array}$

'The sent them down to the big city.' (81)

$\begin{array}{lllll}\text { niyi } & - \text { nhingi } & \text { bal; } & \text { balbirra } & \text {-winmi } \\ \text { that } & -\mathrm{ABL} & \text { ret } & \text { returned } & \text {-they:effected }\end{array}$

'Then they returned.' (82)

$\begin{array}{lllll}\text { ngambirri } & \text {-nyali, } & \text { jilyjarni } & \text {-wirra } & \text { booloomani } \\ \text { again } & \text {-REP } & \text { brand } & \text {-they:extended:him } & \text { bullock }\end{array}$

'Then they branded cattle again.' (83)

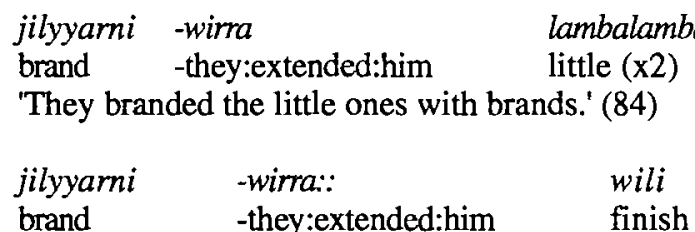

'They branded them, OK.' (85)

barrangga $\quad-y a$

build:up:to:wet -LOC

'In the build up to the wet.' (86)

$\begin{array}{llllll}\text { barrangga } & \text {-barrangga } & \text {-wa } & \text {-wani, } & \text { wila gad } \\ \text { build:up:to:wet } & \text {-build:up:to:wet } & \text {-PROG } & \text {-he:fell } & \text { finish leave } \\ \text {-birrini } & & & & & \\ \text {-they:hit:him } & & & & \end{array}$

'As it gets hot, they leave off work.' (87)

$\begin{array}{llllll}\text { gad } & \text {-birrini } & \text { wimi } & -m i & \text { warang } & \text {-birri } \\ \text { leave } & \text {-they:hit:him } & \text { only } & - \text { next } & \text { sit } & \text {-they:were }\end{array}$

'They left work, and took a break.' (88)

\section{TEXT 3: EARLY CONTACT WITH THE WHITES}

\section{By Bigfoot Jagarra}

[Recorded by William McGregor at Mulurrja, 1982, and transcribed and translated by William McGregor with the assistance of Jack Bohemia, at Fitzroy Crossing, 1982.] ${ }^{7}$

7 In the transcription below WM indicates that the speaker is William McGregor; $X$ indicates another participant in the interaction whose identity is unknown. Everywhere else, the speaker is the narrator. 


\section{GOONTYANDI: EARLY CONTACT WITH WHITES IN THE KIMBERLEY}

doowoo, ngaarriya; doowoo nyamani bagilayi cave, in a hill; there was a big cave

WM: $m h m$ :

niyajiya bagiwirri

$\mathrm{X}$ : aa great (??)

ye:

WM: $m h m$

gamba; goorroomba, thoothooroogbirra windawoorroo goorroomba

$\mathrm{X}: n ? n$ ?

jadimab niyaji

WM: $m h m$

$\mathrm{X}: n ? n$ ?

nd ngaarriya, baabirri, doowooya bagiwirri

$\mathrm{X}$ : thats all

$n ? n$ ?

WM: $n ? n$ ?

garnanganyjanyooloo ngarangaraggilawirri

jajinyooloongarraya barlanyinyooloo

lanngarri

WM: aha

(laughs)

$\mathrm{X:}(\ldots),(\ldots)$

WM: $n h n$

$\mathrm{X}:(\ldots)$

WM: $m h m$

manyi

WM: $m h m$

manyi, wajarri, boongga

$\mathrm{X}$ : ah, all round, (...)

ngabbirra

WM: $m h m$

$n$ ?n?, manyi digooloo, giyimdi, ngabawirri

$\mathrm{X}:(\ldots)$

WM: $n h n$

ye:

$\mathrm{X}$ : all round

WM: aha, ye:

mangarri; mang; manyi mangarri bilawa

WM: $m$ :

marlami

WM: $m h m$

bilawangarrimi bijjangami, wajbali

WM: $n h n$

ngoorndoo ngirnda bijingarni

ngimda girli mamoo bijngami

well wajbalingga kwaitlimjiwina
WM: Mm

they camped there

$X$ : ah great

yes

WM: mm

paper; paperbark, they pulled off paperbark for water (vessel)

$\mathrm{X}: \mathrm{mm}$

they shut it up

WM: $\mathrm{mm}$

$\mathrm{X}: \mathrm{mm}$

and in a hill, inside, in a cave they camped

$\mathrm{X}$ : that's all

$\mathrm{mm}$

WM: $\mathrm{mm}$

what else was it snakes emus and so on they made it on top

WM: aha

(laughter)

$\mathrm{X}$ : (incomprehensible)

WM: mm

$\mathrm{X}$ : (incomprehensible)

WM: $\mathrm{mm}$

vegetable food

WM: $\mathrm{mm}$.

vegetable foods, small potatoes, small potatoes

$\mathrm{X}$ : yes, all around, ...

they ate it

WM: $\mathrm{mm}$

$\mathrm{mm}$, bush plums, and ??, they used to eat

$\mathrm{X}$ : (incomprehensible)

WM: $\mathrm{mm}$

yes

$\mathrm{X}$ : all over the place

WM: um, yes

not; no; not flour food

WM: $\mathrm{mm}$

no

WM: mm

then he may have come with flour, a white man

WM: mm

'who is this who has come up?'

'it's a devil that has come'

well the whites quietened them down 


\section{ABORIGINAL HISTORY 1993 17:1}

right; niyajinhingi, ma:; manyi; niyingga openim, wajbalingga, bilawa ngang

jaji ngirnda::, openimbinmi

yinigawanmanhi, bilawa bidingga openim

right, nyoon, galardingarri

$\mathrm{X}$ : galardi

galardijangi bilawangarri

WM: ye

$\mathrm{X}:(.$.

like $a$ white one

WM: nhnn

clean

WM: yoowayi

nyoonma; garlardingarri, bilawangarringga

WM: aha

$\mathrm{X}:(.$.$) white one$

$m m$

WM: ye

well yinigawanmanhi

WM: $n$;

marlami, labda; ngab; ngaboowarni yan.ginbani, yinigawanmanhi ngaboo

marlami, niyajingga; niyi girli mamoo bijngarni gardganboo

$\mathrm{X:} \mathrm{(...)}$

gadboonggoorroo, bilawa

bidingga briyandi; jinali thalig, nyag; niyaji wajbali, yijgawoo

WM: $n h n$

$\mathrm{X}$ : killim for live

$n::$

WM: $n h n$

(laughter)

boolgawoolga waranggilawirri, (sniff)

wilawoo ngarragi thangarndi niyaji giribli, girrangi winhi

WM: $n h n$ right; then, foo; food; he opened, the white man, and gave (them) flour

'what is it?' they opened (the bags)

'what will we do with it?' (they asked) as they opened the bags of flour

right, (they) rubbed (themselves), (as) with white clay

$\mathrm{X}$ : white clay

with flour like white clay

WM: yes

$\mathrm{X}$ : (indecipherable)

like white stuff

WM: mm

clean

WM: yes

they rubbed themselves; with (like) white clay, with the flour

WM: aha

$\mathrm{X}$ : white stuff

mm

WM: yes

'well what will we do with it?

WM: mm

'nothing, you'll have to; faith; ask your father next, "What will we do with it father?" 'I

'nothing, by this; that's a devil that has come and he might kill us'

$\mathrm{X}$ : (indecipherable)

'leave it alone, that flour'

in turn they; hooked up spears, and speared; that white man, poor bugger

WM: mm

$\mathrm{X}$ : killed him dead

$\mathrm{mm}$

WM: $\mathrm{mm}$

(laughter)

the old men were sitting around

that's all of my story I've finished, it's yours

WM: mm

\section{ORIGINAL TEXT WITH INTERLINEAR TRANSLATIONS}

$\begin{array}{llllllll}\text { doowoo, ngaarri } & -y a ; & \text { doowoo } & \text { nyamani } & \text { bagi } & -l a & -y i \\ \text { cave } & \text { rock } & -\mathrm{LOC} & \text { cave } & \text { big } & \text { lie } & \text {-FACT } & \text {-he:was }\end{array}$

'A cave; there was a big cave in a hill.' (1)

WM: $\operatorname{mhm}:(2)$ 


\section{GOONIYANDI: EARLY CONTACT WTTH WHITES IN THE KIMBERLEY}

niyaji $\quad-y a$ bagi - wimi

this -LOC lie -they:were

'They camped there.' (3)

$\mathrm{X}$ : aa great(??)(4)

ye:

'Yes.' (5)

WM: $m h m(6)$

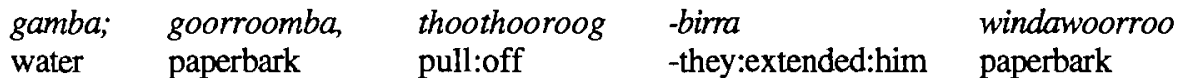
goorroomba

paperbark

'They pulled off paperbark for water (vessel).' (7)

$\mathrm{X}: n ? n$ ?

$\mathrm{X}:$ 'Mm.' (8)

jadimab niyaji

shut this

'They shut it up.' (9)

WM: $m h m(10)$

$\mathrm{X}: n ? n ?$

$\mathrm{X}$ : 'Mm.' (11)

nd ngaarri -ya, baabirri doowoo -ya bagi -wirn and rock -LOC inside cave -LOC lie -they:were

'And in a cave in a hill they campted.' (12)

$\mathrm{X}:$ thats all

$\mathrm{X}$ : 'That's all'. (13

$n ? n ?$

'Mm.' (14)

WM: $n ? n ?$

WM: 'Mm.' (15)

$\begin{array}{llclcc}\text { jaji } & \text {-nyooloo } & \text {-ngarraya } & \text { barlanyi } & \text {-nyooloo gamanganyja } \\ \text { something } & \text {-ETC } & \text {-INCL } & \text { snake } & \text {-ETC } & \text { emu } \\ \text {-nyooloo } & \text { nganangarag } & \text {-gila } & \text {-wirni } & \text { lanngari } \\ \text {-ETC } & \text { make (x2) } & \text {-FACT } & \text {-they:were } & \text { top } \\ \text { 'What else was } & \text { it, snakes, emus and so on, they made it on top.' (16) }\end{array}$




\section{ABORIGINAL HISTORY 1993 17:1}

WM: aha (17)

(laughter)

$\mathrm{X}$ : (Incomprehensible) (18)

WM: $n h n(19)$

$\mathrm{X}$ : (Incomprehensible) (20)

WM: $m h m(21)$

manyi

vegetable:food

'Vegetable food.' (22)

WM: $m h m(23)$

$\begin{array}{lll}\text { manyi, } & \text { wajarri, } & \text { boongga } \\ \text { vegetable:food } & \text { small:potatoes } & \text { small:potatoes }\end{array}$

'Vegetable foods, small potatoes.' (24)

$\mathrm{X}$ : ah, all round, (...) (25)

ngab -birra

eat -they:extended:him

'They ate it.' (26)

WM: $m h m(27)$

$n ? n$ ?, manyi digooloo, giyirndi ngab a

Mm vegetable:food bush:plum ? eat -PROG -they:went

'Mm. They used to eat bush plums and so on.' (28)

$\mathrm{X}$ : (Incomprehensible) (29)

WM: $n h n(30)$

ye:

'Yes' (31)

$\mathrm{X}$ : all round (32)

WM: aha, ye: (33)

$\begin{array}{llll}\text { mangarri; mang; } & \begin{array}{l}\text { manyi } \\ \text { not }\end{array} & \begin{array}{l}\text { mangarri } \\ \text { vegetable:food }\end{array} & \begin{array}{l}\text { bilawa } \\ \text { not }\end{array} \\ \text { 'Not flour.' (34) } & & & \end{array}$


WM: $m:(35)$

marlami

nothing

'No.' (36)

WM: $m h m(37)$

$\begin{array}{lllllll}\text { bilawa } & \text {-ngarri } & -m i & b i j & -j a & -n g a m i, & \text { wajbali } \\ \text { flour } & \text {-COMIT } & \text {-next } & \text { emerge } & \text {-SUBJ } & \text {-it:emerged } & \text { white:people }\end{array}$

'A white man may have come with flour then.' (38)

WM: $n h n(39)$

$\begin{array}{llll}\text { ngoorndoo } & \text { ngimda } & b i j & \text {-ngarni } \\ \text { someone } & \text { this } & \text { emerge } & \text {-it:emerged }\end{array}$

' "Who is this who has come up?" ' (40)

ngimda girli mamoo bij - ngami

this same devil emerge -it:emerged

' "It's a devil that has come." ' (41)

well wajbali $\quad$-ngga kwaitlim $-j i \quad-w i n a$

well white:people -ERG quiet - IT $\quad$-it:extended:to:them

'Well, the whites quietened them down.' (42)

right? niyaji -nhingi, ma:; manyi; niyi -ngga openim, this $-\mathrm{ABL}$ vegetable:food that -ERG

wajbali -ngga, bilawa ngang

white:people -ERG flour give

'Right, then the whites opened (the bags) and gave them flour.' (43)

jaji ngimda::, openim -binmi

something this -they:effected:him

' "What is it?". They opened (the bags).' (44)

yiniga -wanma -nhi, bilawa bidi -ngga openim

do:something -we:pl:will:extend -on:him flour they -ERG

' "What will we do with it?" (they asked), as they opened the bags of flour.' (45)

right, nyoon, galardi -ngarri

rub white:clay -COMIT

'Right, they rubbed themselves (as) with white clay.' (46)

$\mathrm{X}$ : galardi

white:clay

$\mathrm{X}$ : 'White clay.' (47) 


\section{ABORIGINAL HISTORY 1993 17:1}

$\begin{array}{llll}\text { galardi } & \text {-jangi } & \text { bilawa } & \text {-ngarri } \\ \text { white:clay } & \text {-SEM } & \text { flour } & \text {-COMTT }\end{array}$

'With flour, like white clay.' (48)

WM: ye (49)

$\mathrm{X}$ : (Indecipherable) (50)

like a white one

'Like white stuff.' (51)

WM: $n h n(52)$

clean (53)

WM: yoowayi yes

WM: 'Yes.' (54)

nyoon -ma; garlardi -ngarri bilawa -ngarri -ngga rub -now white:clay -COMIT flour -COMT -ERG

'They rubbed themselves with the flour, like white clay.' (55)

WM: aha (56)

$\mathrm{X}:$ (...) white one (57)

$m m$

'Mm.' (58)

WM: ye (59)

well yiniga -wanma -nhi

well do:something -we:pl:will:extend -on:him

" "Well, what will we do with it?" ' (60)

WM: $\mathrm{mm}(61)$

marlami, labda; ngab; ngaboo -wa -mi yan.gin -bani

nothing must father -his -next ask -you:will:fall yiniga -wanma -nhi ngaboo

do:something -we:pl:will:extend -on:him father

' "Nothing. You'll have to ask your father next, "What will we do with it, father"'.' (62)

marlami, niyaji -ngga; niyi girli mamoo bij -ngarni gard nothing this -ERG that same devil emerge -it:emerged hit -ganboo

-he:hits:us

' "Nothing. That's a devil that has come; he might kill us." ' (63) 
$\mathrm{X}:$ (Indecipherable) (64)
gad -boonggoorroo,
bilawa
leave -you:will:hit:it
' "Leave that flour alone." " (65)
flour

$\begin{array}{llllll}\text { bidi -ngga } & \text { briyandi; } & \text { jinali thalig, } & \text { nyag; niyaji } & \text { wajbali; } \\ \text { they -ERG in:turn } & \text { spear hook:up:spear pierce this } & \text { white:people }\end{array}$ yijgawoo

bad

'In turn they hooked up spears and speared that white man, poor bugger.' (66)

WM: $n h n(67)$

$\mathrm{X}$ : killim for live

$\mathrm{X}$ : 'Killed him dead.' (68)

$n::$

Mm. (69)

WM: $n h n(70)$

(laughter)

boolgawoolga warang -gila -wirri, (sniff)

old:men sit -FACT -they:were

'The old men were sitting around.' (71)

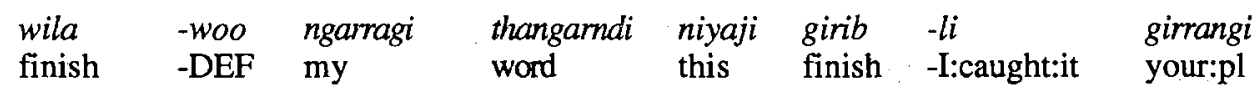
winhi

only

'That's all of my story; I've finished it's yours.' (72)

WM: $\operatorname{nhn}(73)$

\section{PART II}

\section{WILLIAM McGREGOR ${ }^{8}$}

The purpose of this second part to provide some background interpretation for the texts presented above. In particular, I will be engaging in a process of textual exegesis, in an attempt to come to some clearer understanding of what the texts mean, or might mean, to

$8 \quad$ This is a somewhat revised and expanded version of a seminar presentation to the conference 'Aborigines making history', jointly organised by the Australian Institute of Aboriginal Studies and the Australian National University, on 16 May 1988. I am grateful to the participants of the seminar - particularly Eve Fesl, David Nash, Helen Ross - for their comments. I am particularly grateful to Jane Simpson and Peter Read for stimulating comments on an earlier written version of this paper. 
speakers of Gooniyandi, to Gooniyandi people, and what light this might throw on the ways in which they conceptualise the past. I strongly believe that non-Gooniyandi readers of the above Gooniyandi texts will take with them a set of reading practices and cultural baggage which will predispose them to particular readings, which are possibly quite inappropriate, and bear little resemblance to the narrator's intentions. And it is only by a process of exegesis - informed by both a knowledge of the particular language and the culture - that we can come to anything resembling an understanding of the meaning intended by the narrator.

\section{Genre of texts}

I propose that the genre of texts 1 and 2 is a type of exposition, rather than narrative, and that text 3 is perhaps closest to what might be called report. The first thing to notice about the texts is their generality: with a single exception, no attempt is made to establish or identify individual participants, nor are the events alluded to located specifically in time or space. ${ }^{9}$ The texts, in other words, deal in terms of generalities: generic sets (Aborigines and whites), and generic events - in the majority of instances, the events referred to are general classes of events, rather than specific occurrences. ${ }^{10}$ In these terms the three texts contrast sharply with prototypical narratives in Gooniyandi, which as a rule involve both (i) at least one specific and individuated participant, and (ii) beyond the introductory segment providing the setting, a sequence of specific events, which are almost always located in space - that is, at some named place. Perhaps in keeping with this, texts 1 and 2 show a much higher frequency of verbs of cognition and perception than narratives normally do.

Elsewhere ${ }^{11}$ I have argued that narratives in Gooniyandi show the following defining pairs of properties. Firstly, they are structured in terms of one or more STORY UNITS, which contain as inherent constituent elements minimal stories. Minimal stories consist of three conjoined events such that the first and the third are stative, and the second active, with the further property that the third is in some sense an inverse of the first. Secondly, the text as a whole shows the pattern Complication^ ${ }^{\wedge}$ Peripetia^ ${ }^{\wedge}$ enouement, where the Complication represents some kind of disturbance from a previously prevailing inertia, the Peripetia a switch from this disturbed state to the state which prevails finally. The Denouement thus represents not just any stable state, but the resolution of the Complication. The three texts we have been looking at do not, considered as wholes, satisfy these structural descriptions. In particular, the events and states of minimal stories are presumed to be specific and not generics; and the overall organisation into Complication ${ }^{\wedge}$ Peripetia^Denouement does not seem to be satisfied - texts 1 and 3 do not seem to end with a resolution at all; text 2 may, but it is not a resolution of the initial Complication.

How then are the texts then structured?

I would suggest that Text 1 consists of an initial Prologue, which states the topic and authority for the narrator (we return to this later). This is followed by three somewhat narrative like segments (except that they deal with generic events and persons, rather than

9. The exception is the white man Bill Richardson, mentioned on line 57 of text 2 . However, it will be noted that this person does not play a significant role in the unfolding of the text. Once mentioned, the speaker almost immediately forgets him. True, the following clauses have a third person singular Goal (patient or object if you will - see McGregor 1990 for a definition); however, it is by no means clear that he is being specifically referred to, other than in lines 57 and 58 - generic and indefinite Goals are frequently treated as singular for the purposes of pronominal prefixing in Gooniyandi.

Note that in Gooniyandi the past tense alone covers the sense of past habitual or past characteristic.

11 McGregor 1987. 


\section{GOONTYANDI: EARLY CONTACT WITH WHITES IN THE KIMBERLEY}

individuated ones). The first is lines 4 to about 17 ; the second is from about lines 18 to 35 ; and the third is from line 36 to the end. Ignoring the matter of genericity, the first segment begins with the Aborigines in a state of ignorance (line 4), and ends with them in a more knowledgeable condition - see line 14. The second segment begins with Aborigines and whites killing one another in the olden days (lines 18 and 19), and ends with peace and harmony (lines 34 and 35). One might make a case for regarding these as minimal stories, or minimal generic stories, were it not for the fact that the intervening material does not really lead up to the concluding state, but rather merely elaborates on the initial state. The final segment does not seem to have this type of structure at all: it seems to be no more than a report on the particular circumstances, which, though it seems to be clearly regarded as unsatisfactory, is not resolved.

Text 2 is rather similar. It also begins with a type of Prologue outlining the major participants (which are again generic). Then the first narrative like segment begins with a state - that of ignorance of the whites and their ways. It restates this state in a number of variants - ignorance of various different things. There follow, interwoven with the various statements of states of ignorance, a number of events, which culminate in the state of knowledge of line 55. The text then concludes. with a rather lengthy narrative like segment describing life on the stations in the early days. This final segment also seems to be a type of report.

It is noteworthy that the themes of texts 1 and 2 tend to be not the main human participants, but items with which human participants come in contact. ${ }^{12}$ Furthermore, these thematic items are almost everywhere Goals (or patients) in their clauses. In text 2, for instance, there is a sequence of segmented themes (i.e. themes which occur on their own information unit) thiwa 'red' (line $\mathbf{4}$ - note that this does not refer to white people, but attributes a name to them), yawarda 'horse' (line 7), waddgoom 'work' (line 11), manyi 'food' (line 13), and so on. Similar remarks hold for text 2, except that there the themes are not segmented. In both these ways texts 1 and 2 contrast sharply with narratives, which typically have the human protagonists as the sentence themes, and furthermore these thematic protagonists are typically Actors, rather than Goals.

Texts 1 and 2 are apparently dissimilar. However, I suggest that they are each structured as follows. They consist of a sequence of arguments of the form point plus exemplification. Thus, what I have just described as initial state in a type of generic narrative may be alternatively analysed as thesis; the remaining parts of each segment may be seen as evidence in support for this thesis, or elaboration of the thesis - that is, general event sequences are used as evidence for, or elaboration of the general theses. Finally, there is a short concluding piece which states a contrast to the initial thesis; in these texts, it is the now as opposed to the then of the initial thesis. Thus we have a structure something like Thesis^ ${ }^{\wedge}$ Elaboration ${ }^{\wedge}$ Contrast. In the case of Jack Bohemia's texts, the Elaboration also leads into the Contrast in such a way as to provide the intervening causal chain (throughout, it is familiarity through experience and trying the white items and ways); thus text 2 is rather more narrative in character than text 1 , where the contrast is devoid of explanation.

Text 3 seem somewhat different. It clearly falls into two parts, but neither is particularly narrative in the sense of having either minimal story structure, or resolution structure. Each part does no more than relate sequences of generic events; the first comes to no particular conclusion, but merely paves the way for the introduction of the theme - or complication - of the second part. The second part, if it comes to a conclusion at all, it is a

12 The theme - what the utterance is about, or the 'peg' on which it is hinged - in Gooniyandi occurs in initial position in a non-elliptical clause (see McGregor 1990 for detailed discussion). 
very local one, and one which is quite a typical for Gooniyandi narratives - and it certainly does not resolve the complication of this segment. Text 3 could reasonably be regarded as an instance of report. ${ }^{13}$

Putting things another way, these three texts seem to have DESCRIPTION as their primary purpose, rather than the relating of a story with a plot. For this reason it would seem reasonable to group them together as expositions, as distinct from narratives. However, it must be noted that the pieces seem to be merely strung together one after another, and that in each cases, even though there are recurrent themes, there is no clear articulation of a single argument.

I conclude this discussion with brief mention of a couple of observations about the micro-structure of the texts.

Firstly, texts 1 and 2 (but not text 3 ) both begin with brief statements of overall texttopic, which function rather like titles in English writing. Text 1 begins with the word thiwa 'whites', which stands outside of the remainder of the sentence - grammatically, it does not belong to the following clause, and it occurs on its own tone unit. It represents what the story is about - whites, or more accurately, white and Aboriginal interaction. Text 2 begins with a more elaborate statement of topic - 'these people were bush people'. But although this is a full clause, it would seem to be functionally similar to thiwa 'white

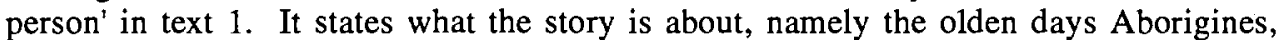
those who lived in the 'bush' - observe that this is the way it is expressed in Gooniyandi, just as in English. Bush people are those who do not live in settled communities; in general they are regarded (by the modern Gooniyandi at least) as rather unsophisticated. A similar, though not identical case might be made for the first word of text 3 , which could be regarded as a synoptic statement of the main theme of the first half of the text: traditional life, typified by the mention of doowoo 'caves'.

Secondly, the three texts do not involve TRAVELLING SEQUENCES; $1{ }^{14}$ that is, they do not have within them sequences of the type 'X left place ${ }_{1}, X$ travelled, $X$ arrived place ${ }_{2}$ '. These sequences are typical of narrative texts, and in part function as markers of authorisation of the text. ${ }^{15}$ Interestingly, there are few indicators of authorisation within these texts; the only really obvious one occurs in the first few lines of text 1 , where Fossil Pluto defers to his jaminyi 'grandfather' (father's father) as his authority for telling the text.

\section{THE TOPICS AND HOW THEY ARE DEALT WITH}

The three texts introduce a number of inter-related themes, some of which recur throughout the texts, while others are mentioned in only one or two of them. These include the following:

(1) Ignorance on the part of early days Aborigines of white people and their ways, and language.

(2) The Aborigines learn white man's ways, and how to deal with new things language, and concepts, etc..

(3) A strong concern with the names given by the early days people for the new items brought by whites.

(4) The contrast then vs. now emerges in each text, though in slightly different ways.

(5) Jack Bohemia stresses the adaptation of Aborigines to white ways, and their crucial role in the cattle industry of the north west.

13 In the sense of, for example, Martin 1985, p.7, and Martin \& Peters 1985.

14 McGregor 1987.

15 ibid. 


\section{GOONIYANDI: EARLY CONTACT WITH WHITES IN THE KIMBERLEY}

(6) Fossil Pluto lays stress on the conflict between black and white, something which is largely ignored by Jack Bohemia.

(7) Fossil Pluto raises the issue of sexual relations between whites and blacks in the last lines of his text.

(8) Fossil Pluto also alludes briefly to ownership of the land. None of the others mention this theme.

Let us look at these themes a little more carefully.

(1) Each text mentions the lack of knowledge on the part of the early days Aborigines of white people, their foods, their artefacts and animals (especially the horse) and their ways of life and language. Consider for instance lines 4 to 9 , and lines 14 to 15 , of text 1 ; lines $2,6,12,13,30$, and 38 of text 2 ; and lines $41,45,55$, and 60 of text 3 . (Note that Jack Bohemia is the only one to mention language difficulties.)

Not only did the Aborigines not know about the introduced items, but also that they either misunderstood them (their nature, etc.) or misused them. Thus for instance, in line 6 of text 1 we find that they thought horses were spirits of dogs, and in line 8 that whites were dwellers of the long grass, presumably some sort of spirit beings. Again, line 41 of text 3 indicates that at first whites were thought to be devils. And again in text 3, lines 45 to 66 deal with flour: it is thought to be white clay, and used as such - they rubbed it on themselves, probably when acting as avengers (who traditionally painted themselves up with white clay before going on revenge expeditions). This would seem to best explain line 66.) Although Jack Bohemia's text does not mention this misunderstanding about flour, he was well aware of it, and commented on it himself on other occasions. He also mentioned that tea was thought to be gri, a type of sap used to poison fish - gri turns water to a redish colour like tea. Thus the early days Aborigines thought that the whites were trying to poison them.

Ignorance, it will also be noted, is advanced as perhaps the main reason for conflict. See text 1 , lines $18-19,21$, and 29.

(2) The second theme - that Aborigines learnt white man's ways, and how to deal with new things language, and concepts, etc. - is the major theme of text 2, by Jack Bohemia. It is briefly alluded to in text 1 (see lines 14 and 29-30), where Pluto merely says that whereas before people were ignorant, now they are not; text 3 seems to ignore it completely.

(3) Texts 1 and 2 both mention the names given by early days people for the new items brought by whites. Lines 6 and 8 of text 1 indicate that horses were first called thiminyjangarri, 'spirits of dogs' and marrimbala 'grass country devil'. (One of the common words for 'horse', dimana, apparently also had this original meaning in Gooniyandi, according to information from Jack Bohemia.) Line 8 also indicates that whites were called gilimboormgarnanyi, 'dwellers of the long grass'. Jack Bohemia also cites marrimbala (and a variant maroonbala) as the early name for the horse (see lines 8 and 9). However, Bohemia says (in line 3) that thiwa was the first name given to whites - whether or not this is true, it certainly is the word that most Gooniyandi speakers identify as the Gooniyandi word for 'white person'. Bohemia also gives the words for two other introduced items: baarri 'wagon', booloomani 'cattle' (see lines 40 and 41 respectively).

This is very interesting information for the historical linguist, as it is possible on the basis of such information to establish different time periods of different linguistic adaptations to the white society. We can trace, for instance, the following changes over time, with respect to some of the more common introduced items: 
ABORIGINAL HISTORY 1993 17:1

\begin{tabular}{|c|c|c|c|}
\hline Meaning & First form: & $\frac{\text { 'Received' }}{\text { Gooniyandi }}$ & Forms in use today \\
\hline $\begin{array}{l}\text { 'white person' } \\
\text { 'horse' }\end{array}$ & $\begin{array}{l}\text { gilimboormgarnanyi } \\
\text { thiminyjangarri, }\end{array}$ & thiwa & malngarri, thiwa, gardiya, wajbali \\
\hline 'policeman' & $?$ & $\begin{array}{l}\text { mirnmirdgali } \\
\text { mirna }\end{array}$ & limba' \\
\hline
\end{tabular}

These two texts would seem to closely associate naming with understanding, such that knowing the correct name is identifiable with understanding, while having an incorrect name is tantamount to misunderstanding. Thus the early names for white people and horses reflect the early misunderstandings about the nature of these novelties. Both were presumed to be from the spirit realm. In a similar way, flour was understood to be white clay, which put it in the world of retribution, law, rather than food. These early names gave way to names which reflected a changed understanding of the introduced item. Thus, whites were not understood any longer as spirits, and so were named after a physical attribute, the colour of their skin. The term dimana 'horse' was one used over a wide area extending as far as the Queensland border. ${ }^{16}$ The final column reflects the modern linguistic situation, in which there has been a shift from speaking Gooniyandi firstly to speaking Walmajarri as a lingua franca amongst Fitzroy Crossing Aborigines, followed by a shift to speaking Kriol. Today, the most common and frequent terms for introduced items are pan-Kimberley, and not the ones speakers associate specifically with particular languages.

(4) The contrast between then and now emerges in slightly different ways in the three texts. Fossil Pluto contrasts the violence of the frontier with the relative peace of today (line 35 of text 1). Jack Bohemia (text 2) contrasts the ignorance of the past with respect to whites and white artefacts etc. with the knowledge of the present. (This is a view he holds independent of this particular text - that knowledge of whites and their ways is important, and a necessary prerequisite for everyone who lives in the modern world.) Thirdly, Bigfoot Jagadda contrasts the 'then' of the traditional past with the 'now' of the period of first contact (rather than the now of the present). Thus relative calm is imputed to the traditional life, where people lived and ate (lines 1 through to about 35).

(5) The adaptation of Aborigines to white ways, and their crucial role in the cattle industry of the North West is stressed by Jack Bohemia. Thus the second half of Bohemia's text (lines 51 onwards) deals with stockwork. First, in lines 51 to 54 , we have the Aborigines learning to ride horses as the first thing they learn about stockwork. Then in lines 57 to the end of the text, there is a piece about stockwork, and the various jobs it entails. The Bill Richardson of line (57) was the first manager of Old Bohemia station, on which Bohemia was born and where he first worked for many years. (It was Bohemia's belief that this was the first station in the region; however, written historical sources disagree and place Fossil Downs as the first in the region.) This segment (lines 57 to the end) describes how Aborigines learnt to look after ('grow up') the cattle (line 60), brand them (line 62-63), and make cattle yards (lines 64-69). In lines 77 to 83 we have the Aborigines driving the cattle to Derby, to be put on the boats - thus there were Aborigines who were entrusted with jobs requiring responsibility, diligence and ability to work without supervision. It was not the case that, as is often said, the Aborigines had to be supervised all the time to ensure they worked. ${ }^{17}$

16 Simpson 1985 , p. 14

17 See also Shaw 1986; McGrath 1987; Marshall 1989. 


\section{GOONIYANDI: EARLY CONTACT WITH WHITES IN THE KIMBERLEY}

The final three lines of the text allude to the practice in the north of Australia of not working during the wet season (roughly December to April). Aboriginal employees were normally free to 'go bush' at this time.

The opinions expressed here are of a man who has worked all of his life in white employment, first as a stockman and later as a police tracker. Indeed, he excelled in each to the extent that he is regarded as amongst the best stockmen of the Kimberley region (by younger Aboriginal men whom he taught), and the best tracker of the north-west. Although the other two narrators also presumably worked on the stations, their major claim to fame is as lawmen, and they do not mention stockwork.

(6) The conflict between black and white is a recurrent theme in Fossil Pluto's text. However, it is by no means a one-sided battle. Lines $11,13,18$, and 28 all refer to Aborigines killing whites. In fact, the first violent act is attributed to Aborigines. Lines $18,19,22,25,27$, and 31 refer to whites killing Aborigines, or their propensity to do so. Conflict is, however, largely ignored by Jack Bohemia and Bigfoot Jagadda. In fact, it is not mentioned at all by Bohemia, and Bigfoot mentions it only indirectly, in line 42 of his contribution (text 3 ), by the allusion to 'quietening down'. And again in line 66, except that this time it is Aborigines spearing a white man.

(7) Miscegenation is the final issue mentioned in Fossil Pluto's text. The theme is first mentioned in line 26, but the speaker then goes on to talk about fighting and killing, not returning to this theme again until line 37 (or possibly 36 - the meaning of this line (like line 38) is by no means clear). The results of these liaisons - 'part Aborigines' - are also mentioned.

It might be noted here that Pluto presents it not as rape by white men, but that the Aboriginal women went sneekingly to the white men. (There is, however, some equivocation, or at least duality in his thought: the first mention puts it as whites stealing Aboriginal women.) In other words, on this issue, as for the others, the Gooniyandi speaker has not presented Aborigines as prototypical patients, whites as prototypical agents. In fact, a strong characteristic of the three texts is that they portray Aborigines as ACTORS. They are not the mere passive recipients of white violence, but also contributed themselves to the violence of the frontier - not, it should be noted, as a form of resistance.

The other recurrent theme, which is perhaps an extension of this theme of Aborigines as actors, is learning and cooperation; they adapted to the new world in such a way as to retain their agentive potency.

(8) Finally, only Fossil Pluto alludes, albeit briefly, to the issue of ownership of the land. He merely states in line 20 that whites tramped over Aboriginal land, and this is given as one of the reasons for conflict (the other being ignorance, as has already been mentioned). Thus, in the context of these texts, there seem to be no strong arguments presented for exclusive Aboriginal ownership of the land.

\section{Relation to other stories of early contact with whites in the Kimberley and elsewhere}

These three Gooniyandi texts are not untypical of stories of the early contact with whites and there are available similar stories from other Aboriginal groups that deal with some of the same, or a set of very similar, basic elements. I have recorded quite similar texts in other Kimberley languages, including Unggumi, Bunuba, and Aboriginal English; and Helen Ross (pers. comm.) reports that she has collected similar texts in Kriol, Aboriginal English and Kija at Turkey Creek. Published texts and text portions include Thoorrbiliny (1987), McGrath (1987), Nathan and Japanangka (1983), and Moses and Tsunoda (1986), amongst many others. 
Nathan and Japangangka ${ }^{18}$ include a piece on early contact, which forms part of a larger text told by a group of Warlpiri women to Pam Nathan (in Warlpiri), a translation of which appears on pages 47 to 50 of that book. (It may be relevant that neither author is a linguist, and the story was not collected primarily for the purposes of linguistic investigation.) That text falls roughly into two parts: the first, approximately three-quarters, deals with traditional lifestyle; the final quarter mentions contact. Specific issues mentioned are: (a) Aborigines' lack of clothing, which is overcome with gifts from whites; (b) ignorance of the white man; (c) trying white man's food (in this instance, it is beef that they try first, flour later); and (d) wondering where the whites come from (compare line 5 of text 2 and line 40 of text 1 ). However, the contrast between then and now is largely absent and naming practices are not mentioned. Likewise, Thoorrbiliny ${ }^{19}$ deals mainly with the initial conflict, and its later resolution, but also briefly deals with the theme of the Aborigines' ignorance of white foods, and their later control of white foods, artefacts, and institutions (such as riding, branding cattle, and so on). Thoorrbiliny's text is also a part of a larger text, one with a political purpose. Here the past is contrasted with the present, and the text about the early contact is used partly as an argument for Aboriginal control of some tracts of land. The summary and excerpts of a similar piece by the Gurindji woman, Amy Laurie, in McGrath ${ }^{20}$ deals with (a) mistaken beliefs about whites and horses - that they were devils; (b) conflict and massacres; (c) bringing cattle and quietening the people; (d) miscegenation; (e) and introduction of white foods.

The other themes remarked on above, Aborigines as actors, and cooperation recur throughout these texts.

Some accounts of first contact are, however, rather different, in that they are predominantly focussed on a single issue, that of the violent confrontation between whites and Aborigines. For instance, the Jaru story by Robert Moses ${ }^{21}$ relates just one issue, the violent confrontation between cattlemen and Aborigines over access to water.

One very striking difference between the three Gooniyandi stories and the other accounts alluded to above is that the latter ${ }^{22}$ all mention massacres, using these as the main illustrations of white-Aboriginal conflict. The Gooniyandi stories do not refer to specific massacres, although they do refer generically to massacres or large scale killings. That is, they take no single instance as an exemplar for the violence of the frontier. Indeed, as we have seen, they place as much weight on Aboriginal violence as they do on white violence.

It is interesting that, like Jack Bohemia, Moses and Thoorrbiliny, who were recorded by linguists, both mention language. Both discuss difficulties of communication due to the lack of a common language, and use this as part of the explanation of the conflict.

There are some interesting similarities and differences amongst the additional texts we have mentioned in this section in terms of their organisational patterns. As is the case for the Gooniyandi texts, there is neither detailed temporal location, nor spatial succession. Although events are in some instances given spatial locations, what is absent is the detailed place to place movement characteristic of most narrative genres in Aboriginal languages. I suspect that there may be reasons for this; in all areas dealt with, the events fall out of living memory, and probably also outside of the living memory of the generation before the speaker. (In this context, it would be interesting to compare stories from people whose contact has been more recent - e.g. the Desert people who arrived in Fitzroy Crossing in the

Nathan \& Japanangka 1983, pp.49-50.

Thoortbiliny 1987.

McGrath 1987, pp.1-2.

Hercus \& Sutton 1986, pp.41-46.

Including Thoorrbiliny 1987; Moses \& Tsunoda 1986; McGrath 1987. 


\section{GOONIYANDI: EARLY CONTACT WTTH WHITES IN THE KIMBERLEY}

mid 20th century.) Thus we might expect a significant reduction in the details remembered of the early contact stories that speakers of today may have heard as children.

In some instances the stories have the overall structure of narratives; they do not use narrative segments as parts of illustrations of more general points, as in the case of the Gooniyandi texts. Examples of this type include Moses' contribution in Hercus and Sutton, ${ }^{23}$ and Laurie's in McGrath. ${ }^{24}$ One wonders whether this generic difference might be relatable to 'external' factors such as the temporal distance from initial contact, and the nature of the actual contact: whether, for instance, it was characterised by conflict over a long period of time, with numerous events of various types, or whether it was typified by single particularly violent events, which have been inter interpreted as standing for, as exemplary examples of, the frontier of conflict.

\section{Conclusion}

In this part of the paper I have discussed the three Gooniyandi texts presented in Part I, identifying themes and the ways in which they are dealt with. I have also briefly compared these texts with texts on similar themes from other Australian groups. My main purpose has been to draw attention to these texts as statements by Aborigines. At the same time, it is my interpretation presented here, and I have been involved in all phases of the production of the Gooniyandi texts. The texts are not 'pure' in any sense, as statements by Gooniyandi people 'untainted' by white influence. The need for exegetical investigations such as this is, firstly, that when the cultures are as different as Aboriginal and white there is room for misunderstanding and confusion on each side; and secondly, related to this, in the context of today we need to examine 'original' source materials - or what is as close as possible to original materials - not merely those which have been put through the mill of a historian's interpretation. To put it another way, this is one relatively small aspect of a number of larger projects I am currently working on whose aim is to present effectively Gooniyandi views of past events, rather than to use these texts like documents in a library, as fields to be mined for evidence to support my own theories of Kimberley history. More generally, it is a call for the development of new genres for talking about the past.

The three texts are about the past, and make arguments about the past. But we should still raise the question: are they historical? It is by no means clear that every text dealing with the past should qualify as a historical text (even though it may contain historical information). The vast majority of Gooniyandi texts I have collected about the past are quite different to the three which have been the focus of this paper. They are narrative in form, and specific and individuated in both participant (character) and event reference. Moreover, they incorporate an ideology of the past which relates past events and happenings to PLACES, rather than times, and for this reason the term historical is at best misleading. Our three texts are neither narrative nor place orientated. Nor are they time orientated. The fact that they present arguments and elaboration of theses makes them resemble modern history (whose essential character has been described by Legge ${ }^{25}$ as argumentative, and defending a point of view), perhaps more than Gooniyandi narratives of the past. Differences must, however, be kept in mind. Unlike modern history, these texts deal almost exclusively in generalities, and do not refer to particular events of the past.

\footnotetext{
23 Hercus \& Sutton 1986

24 McGrath 1987, pp. 1-2.

25 Legge 1988.
} 


\section{ABORIGINAL HISTORY 1993 17:1}

\section{LIST OF REFERENCES}

Bohemia, J. \& McGregor, W.B. 1991, 'Death practices in the North West of Australia', Aboriginal History, 15, pp.86-106.

Hercus, L. \& Sutton, P. (eds) 1986, This is What Happened: Historical Narratives by Aborigines, Canberra.

Legge, J. 1988, Perspectives on the past, plenary paper presented to Conference 'Aborigines making history', Canberra, 16 May.

Marshall, P. 1989, Raparapa: All Right, Now We Go 'longside the River: Stories from the Fitzroy River Drovers, Broome, W.A.

Martin, J. 1985, Factual Writing: Exploring and Challenging Social Reality, Geelong, Vic.

Martin, J. \& Peters, P. 1985, 'On the analysis of exposition', in Discourse on Discourse, ed. R. Hasan, Applied Linguistics Association of Australia, Occasional Papers, no.7, pp.61-92.

McGrath, A. 1987, Born in the Cattle, Sydney.

McGregor, W.B. 1987, 'The structure of Gooniyandi narratives', Australian Aboriginal Studies, 1987, no.2, pp.20-8.

1988, 'Jack Bohemia and the Banjo affair', Meridian, vol.7, no.1, pp.34-58. 1990, A Functional Grammar of Gooniyandi, John Benjamins, Amsterdam.

Moses, R. \& Tsunoda, T. 1986, 'The first white man comes to Nicholson River', in This is What Happened: Historical Narratives by Aborigines, eds L. Hercus \& P. Sutton, Canberra, pp.41-6.

Nathan, P. \& Japanangka, D.L. 1983, Health Business, Heinemann, Melboume.

Shaw, B. 1986, Countrymen: The Life Histories of Four Aboriginal Men as Told to Bruce Shaw, Canberra.

Simpson, J. 1985, 'How Warumungu people express new concepts', Language in Central Australia, 4, pp.12-25.

Thoorrbiliny, R. 1987, The effect of white land use on the Miriwoong people, transcribed by F. Kofod, manuscript of paper presented to Conference 'Aborigines and development in the East Kimberley', Kununurra, WA, 11-13 May. 